\title{
DISCRETE SERIES CHARACTERS AND TWO-STRUCTURES
}

\author{
REBECCA A. HERB
}

\begin{abstract}
Let $G$ be a connected semisimple real Lie group with compact Cartan subgroup. Harish-Chandra gave formulas for discrete series characters which are completely explicit except for certain interger constants appearing in the numerators. The main result of this paper is a new formula for these constants using two-structures. The new formula avoids endoscopy and stable discrete series entirely, expressing (unaveraged) discrete series constants directly in terms of (unaveraged) discrete series constants corresponding to two-structures of noncompact type.
\end{abstract}

\section{INTRODUCTION}

Let $G$ be a connected semisimple real Lie group with finite center. Associated to each irreducible unitary representation $\pi$ of $G$ is its distributional character $\Theta_{\pi}$. These characters are actually analytic functions on the regular set of $G$, and it is a fundamental problem of harmonic analysis to find formulas for these characters as functions on the regular set. For the important class of tempered representations these character formulas are well understood. The fundamental representations in this case are the irreducible discrete series (and limit of discrete series) representations. All other irreducible tempered representations can be obtained from these representations via unitary parabolic induction [K-Z]. Since character formulas for parabolically induced representations are known, given the formula for the inducing representation, the basic objects of study are the characters of discrete series representations.

Harish-Chandra gave formulas for discrete series characters in [HC1]. These formulas are completely explicit except for certain integer constants appearing in the numerators. The main result of this paper is a new formula for these constants. This formula uses the notion of a two-structure introduced in [H3] to give a formula for the analogous constants appearing in formulas for averaged discrete series characters. A more complicated formula for the (unaveraged) discrete series constants was given in [H4]. There the idea of endoscopy was used to write formulas for discrete series constants in terms of averaged constants for endoscopic groups. The new formula avoids endoscopy entirely, expressing discrete series constants directly in terms of (unaveraged) discrete series constants corresponding to two-structures of noncompact type. It could be used to greatly simplify the proof of the Fourier inversion formulas for orbital integrals in [H4]. It is not clear how two-structures

Received by the editors April 8, 1996 and, in revised form, October 4, 1996.

1991 Mathematics Subject Classification. Primary 22E30, 22E45.

Supported by NSF Grant DMS 9400797 and a University of Maryland GRB Semester Research Grant. 
are related to the more geometric formulas for discrete series and averaged discrete series constants in [G-K-M].

In its simplest form the result can be stated as follows. Let $\Phi$ be a root system in a real vector space $E$. We assume that $\Phi$ contains a root system $\Phi_{K}$, called the compact roots, and that $\Phi$ is spanned by a set of strongly orthogonal noncompact roots. Then we have a discrete series constant $d\left(E^{*}: \Phi^{+}\right)$for each choice $\Phi^{+}$of positive roots and each connected component $E^{*}$ of

$$
E^{\prime}=\{\tau \in E:\langle\tau, \alpha\rangle \neq 0 \forall \alpha \in \Phi\} .
$$

In [H3] we defined a set $\mathcal{T}(\Phi)$ of two-structures for $\Phi$, and associated to each $\phi \in \mathcal{T}(\Phi)$ and choice $\Phi^{+}$of positive roots a sign $\epsilon\left(\phi: \Phi^{+}\right)= \pm 1$. Each $\phi \in \mathcal{T}(\Phi)$ is a root system contained in $\Phi$, has the same rank as $\Phi$, and has all irreducible factors of type $A_{1}$ or $B_{2} \simeq C_{2}$. Let $\phi_{K}=\phi \cap \Phi_{K}$. We say a two-structure is of noncompact type if it is spanned by a strongly orthogonal set of noncompact roots, and write $\mathcal{T}_{n}(\Phi)$ for the set of all two-structures of noncompact type. Let $\Phi^{+}$be a choice of positive roots for $\Phi$ and let $E^{*}$ be a connected component of $E^{\prime}$. Then for each $\phi \in \mathcal{T}_{n}(\Phi), \phi^{+}=\phi \cap \Phi^{+}$gives a choice of positive roots for $\phi$, and there is a unique connected component $E_{\phi}^{*}$ of

$$
E^{\prime}(\phi)=\{\tau \in E:\langle\tau, \alpha\rangle \neq 0 \forall \alpha \in \phi\}
$$

which contains $E^{*}$. This data parameterizes a discrete series constant $d\left(E_{\phi}^{*}: \phi^{+}\right)$. Since every irreducible factor of $\phi$ is of type $A_{1}$ or $B_{2}, d\left(E_{\phi}^{*}: \phi^{+}\right)$is a product of discrete series constants corresponding to root systems of type $A_{1}$ or $B_{2}$. These constants are easy to compute. (See for example [K, p. 498].) Define $c(\Phi)=$ $[L(\phi): L(\Phi)]$, the index of the weight lattice of $\Phi$ in the weight lattice of $\phi$. It is independent of $\phi \in \mathcal{T}_{n}(\Phi)$, since all two-structures are conjugate via the Weyl group of $\Phi$. The main result of this paper is the following theorem, which will be stated in a more detailed form as Theorem 3.4 below.

Theorem. Let $\Phi^{+}$be a choice of positive roots for $\Phi$ and let $E^{*}$ be a connected component of $E^{\prime}$. Then

$$
d\left(E^{*}: \Phi^{+}\right)=c(\Phi) \sum_{\phi \in \mathcal{T}_{n}(\phi)} \epsilon\left(\phi: \Phi^{+}\right) d\left(E_{\phi}^{*}: \phi^{+}\right) .
$$

The theorem is proven by showing that the right hand side of the equation has the usual properties which can be used to characterize discrete series constants. The only one of these which is difficult to verify is the patching condition.

\section{Discrete Series Character Formulas}

Let $G$ be a connected semisimple Lie group with a compact Cartan subgroup $B$. For any closed subgroup $H$ of $G$, we denote the real Lie algebra of $H$ by the corresponding lower case German letter $\mathfrak{h}$ and the complexification of $\mathfrak{h}$ by $\mathfrak{h}_{\mathbf{C}}$. Let $K$ be a maximal compact subgroup of $G$ containing $B$ and let $\mathfrak{g}=\mathfrak{k}+\mathfrak{p}$ be the corresponding Cartan decomposition.

Let $\Phi_{G}=\Phi\left(\mathfrak{g}_{\mathbf{C}}, \mathfrak{b}_{\mathbf{C}}\right)$ denote the roots of $\mathfrak{b}_{\mathbf{C}}$ in $\mathfrak{g}_{\mathbf{C}}$, and let $\Phi_{K}=\Phi\left(\mathfrak{k}_{\mathbf{C}}, \mathfrak{b}_{\mathbf{C}}\right)$ denote the set of compact roots. For any root system $\Phi \subset \Phi_{G}$, we will let $W(\Phi)$ denote the Weyl group generated by reflections in roots of $\Phi$. Since $G$ is connected, $W_{G}=N_{G}(B) / B$ can be identified with $W\left(\Phi_{K}\right)$. 
Let $E=i \mathfrak{b}^{*}$ denote the real vector space of all complex linear functionals on $\mathfrak{b}_{\mathbf{C}}$ which take pure imaginary values on $\mathfrak{b}$. The $\Phi_{G} \subset E$ and we define

$$
E^{\prime}=\left\{\tau \in E:\langle\tau, \alpha\rangle \neq 0 \forall \alpha \in \Phi_{G}\right\} .
$$

For any root system $\Phi \subset \Phi_{G}$, we also define

$$
E^{\prime}(\Phi)=\{\tau \in E:\langle\tau, \alpha\rangle \neq 0 \forall \alpha \in \Phi\} .
$$

Fix a choice of positive roots $\Phi_{G}^{+}$and let $\rho$ be half the sum of the roots in $\Phi_{G}^{+}$. Then $\lambda \in E^{\prime}$ is called a discrete series parameter for $G$ if $e^{\lambda-\rho}$ gives a well-defined character of $B$.

Fix a discrete series parameter $\lambda \in E^{\prime}$. Write

$$
\Delta_{B}(b)^{\prime}=\prod_{\alpha \in \Phi_{G}^{+}}\left(1-e^{-\alpha}(b)\right), \quad b \in B
$$

and define

$$
q=\frac{1}{2} \operatorname{dim} G / K, \quad \epsilon(\lambda)=\operatorname{sign} \prod_{\alpha \in \Phi_{G}^{+}}\langle\alpha, \lambda\rangle .
$$

Then, by $[\mathrm{HC} 2, \S 27]$ there is a unique discrete series representation of $G$ with character $\epsilon(\lambda)(-1)^{q} \Theta_{\lambda}$, where $\Theta_{\lambda}$ is given on regular elements $b \in B$ by

$$
\Delta_{B}(b)^{\prime} \Theta_{\lambda}(b)=\sum_{w \in W_{G}} \operatorname{det} w e^{w \lambda-\rho}(b) .
$$

To describe the formula for $\Theta_{\lambda}$ on noncompact Cartan subgroups, we follow the notation of Harish-Chandra in [HC1, $\S 22]$. Thus we let $A$ denote a $\theta$-stable Cartan subgroup of $G$ with $A=A_{I} A_{R}, A_{I}=A \cap K, \mathfrak{a}_{R} \subset \mathfrak{p}$. Fix a connected component $A^{+}$of

$$
A^{\prime}(R)=\left\{h \in A: e^{\alpha}(h) \neq 1 \forall \alpha \in \Phi_{R}\left(\mathfrak{g}_{\mathbf{C}}, \mathfrak{a}_{\mathbf{C}}\right)\right\},
$$

where $\Phi_{R}\left(\mathfrak{g}_{\mathbf{C}}, \mathfrak{a}_{\mathbf{C}}\right)$ denotes the set of roots of $\mathfrak{a}_{\mathbf{C}}$ in $\mathfrak{g}_{\mathbf{C}}$ which take real values on $\mathfrak{a}$. Then $A^{+}=A_{I}^{+} A_{R}^{+}$, where $A_{I}^{+}$is a connected component of $A_{I}$ and $A_{R}^{+} \subset A_{R}$. We can assume (by conjugating if necessary) that $A_{I}^{+} \subset B$. Let $\mathfrak{z}$ be the centralizer of $A_{I}^{+}$in $\mathfrak{g}$, and let $\Xi$ be the analytic subgroup of $G$ corresponding to $\mathfrak{z}$. Then $\mathfrak{b}$ and $\mathfrak{a}$ are both Cartan subalgebras of $\mathfrak{z}$. Fix a Cayley transform $y \in A d(\mathfrak{z} \mathbf{C})$ such that $y\left(\mathfrak{b}_{\mathbf{C}}\right)=\mathfrak{a}_{\mathbf{C}}$. Then $y \Phi_{G}^{+}$gives a choice of positive roots for $\Phi\left(\mathfrak{g}_{\mathbf{C}}, \mathfrak{a}_{\mathbf{C}}\right)$. For $h \in A$, define

$$
\Delta_{A}(h)^{\prime}=\prod_{\alpha \in y \Phi_{G}^{+}}\left(1-e^{-\alpha}(h)\right) .
$$

If $G$ is acceptable we can also define

$$
\Delta_{A}(h)=e^{y \rho}(h) \Delta_{A}(h)^{\prime} .
$$

Write $\Phi=\Phi\left(\mathfrak{z}_{\mathbf{C}}, \mathfrak{b}_{\mathbf{C}}\right)$. Since $\mathfrak{z}$ has a Cartan subalgebra $\mathfrak{a}$ which is split modulo the center of $\mathfrak{z}$, the root system $\Phi$ is spanned by a strongly orthogonal collection of noncompact roots. Further, $y \Phi \subset \Phi_{R}\left(\mathfrak{g}_{\mathbf{C}}, \mathfrak{a}_{\mathbf{C}}\right)$. Let $W\left(A^{+}\right)=W(y \Phi), W\left(A_{I}^{+}\right)=$ $W_{G} \cap W(\Phi)$, and $W_{\Xi}=N_{\Xi}(B) / B$. Note that $W_{\Xi}$ is a subgroup of $W\left(A_{I}^{+}\right)$. 
Assume that $G$ is acceptable. Then by [HC1, Lemma 59], for each $s \in W\left(A^{+}\right)$ and connected component $E^{*}$ of $E^{\prime}$ there is a unique constant $c_{H C}\left(s: E^{*}: A^{+}\right)$, such that for all $h_{1} \in A_{I}^{+}, h_{2} \in A_{R}^{+}$,

$$
\begin{gathered}
\Delta_{A}\left(h_{1} h_{2}\right) \Theta_{\lambda}\left(h_{1} h_{2}\right)=\sum_{t \in W\left(A_{I}^{+}\right) \backslash W_{G}} \operatorname{det} t e^{t \lambda}\left(h_{1}\right) \\
\times \sum_{s \in W\left(A^{+}\right)} \operatorname{det} s c_{H C}\left(s: t E^{*}(\lambda): A^{+}\right) \exp \left(s(t \lambda)^{y} \log h_{2}\right) .
\end{gathered}
$$

Here $E^{*}(\lambda)$ is the connected component of $E^{\prime}$ containing $\lambda$.

For any $s \in W\left(A^{+}\right)$and connected component $E^{*}$ of $E^{\prime}$, by [HC1, Lemma 58] we have

$$
c_{H C}\left(s: E^{*}: A^{+}\right)=\sum_{t \in W\left(A_{I}^{+}\right) / W \Xi} c_{\mathfrak{z}}\left(s t^{y}: t^{-1} E^{*}: \mathfrak{a}^{+}\right)
$$

where $\mathfrak{a}^{+}=\mathfrak{a}_{I}+\mathfrak{a}_{R}^{+}$, and the constants $c_{\mathfrak{z}}\left(s: E^{*}: \mathfrak{a}^{+}\right)$come from the theory of the Lie algebra [HC1, Lemma 40]. Using the definition following [HC1, Lemma $40]$, we see that $c_{\mathfrak{z}}\left(s: E^{*}: \mathfrak{a}^{+}\right)$depends only on the connected component $E_{\mathfrak{z}}^{*}$ of $E^{\prime}(\Phi)$ containing $E^{*}$. Formula $(2.3)$ shows that the constants $c_{H C}\left(s: E^{*}: A^{+}\right)$ depend only on the local isomorphism class of $G$, and are given by a simple formula in terms of constants which depend only on data corresponding to $\mathfrak{z}$, not on the original group $G$.

As in the proof of Lemma 59 in the middle of page 304 of [HC1], we can rewrite equation (2.2) in terms of these local constants as

$$
\begin{gathered}
\Delta_{A}\left(h_{1} h_{2}\right) \Theta_{\lambda}\left(h_{1} h_{2}\right)=\sum_{t \in W \Xi \backslash W_{G}} \operatorname{det} t e^{t \lambda}\left(h_{1}\right) \\
\times \sum_{s \in W\left(A^{+}\right)} \operatorname{det} s c_{\mathfrak{z}}\left(s: t E^{*}(\lambda): \mathfrak{a}^{+}\right) \exp \left(s(t \lambda)^{y} \log h_{2}\right) .
\end{gathered}
$$

Note that $W\left(A^{+}\right)=W(y \Phi)=\left\{w^{y}: w \in W(\Phi)\right\}$. Further, if we define

$$
\Phi\left(\mathfrak{a}^{+}\right)=\left\{\alpha \in \Phi: y \alpha(H)>0 \forall H \in \mathfrak{a}^{+}\right\},
$$

then $\Phi\left(\mathfrak{a}^{+}\right)$is a choice of positive roots for $\Phi$, and $\mathfrak{a}^{+}$is the positive Weyl chamber of $\mathfrak{a}$ with respect to $y \Phi\left(\mathfrak{a}^{+}\right)$. We change notation slightly and write

$$
c\left(w: E^{*}: \Phi\left(\mathfrak{a}^{+}\right)\right)=c_{\mathfrak{z}}\left(w^{y}: E^{*}: \mathfrak{a}^{+}\right) .
$$

Then we can rewrite equation (2.4) as

$$
\begin{gathered}
\Delta_{A}\left(h_{1} h_{2}\right) \Theta_{\lambda}\left(h_{1} h_{2}\right)=\sum_{t \in W \Xi \backslash W_{G}} \operatorname{det} t e^{t \lambda}\left(h_{1}\right) \\
\times \sum_{w \in W(\Phi)} \operatorname{det} w c\left(w: t E^{*}(\lambda): \Phi\left(\mathfrak{a}^{+}\right)\right) \exp \left((w t \lambda)^{y} \log h_{2}\right) .
\end{gathered}
$$

Since the Cayley transform $y$ centralizes $A_{I}^{+}$,

$$
\Delta_{A}\left(h_{1} h_{2}\right)=e^{y \rho}\left(h_{1} h_{2}\right) \Delta_{A}\left(h_{1} h_{2}\right)^{\prime}=e^{\rho}\left(h_{1}\right) e^{y \rho\left(\log h_{2}\right)} \Delta_{A}\left(h_{1} h_{2}\right)^{\prime} .
$$


Thus we can rewrite formula (2.5) as

$$
\begin{gathered}
\Delta_{A}\left(h_{1} h_{2}\right)^{\prime} \Theta_{\lambda}\left(h_{1} h_{2}\right)=\sum_{t \in W_{\Xi} \backslash W_{G}} \operatorname{det} t e^{t \lambda-\rho}\left(h_{1}\right) \\
\times \sum_{w \in W(\Phi)} \operatorname{det} w c\left(w: t E^{*}(\lambda): \Phi\left(\mathfrak{a}^{+}\right)\right) \exp \left((w t \lambda-\rho)^{y} \log h_{2}\right) .
\end{gathered}
$$

Now suppose that $G$ is not acceptable and $\lambda$ is a discrete series parameter for $G$. As in [HC2, §27], $G$ has a two-fold acceptable cover $G_{1}$, and $\lambda$ is also a discrete series parameter for $G_{1}$ with character formula (2.6). But since both sides of equation (2.6) are well-defined on $G$, the formula is also valid for the discrete series character formula on $G$.

Remark. The assumption that $G$ has finite center is not really necessary. Using the techniques in $[\mathrm{H}-\mathrm{W}]$, formula (2.6) can also be used in the case that $G$ has infinite center and $B$ is compact modulo the center of $G$. In this case the representations are not true discrete series, but rather "relative discrete series".

We want to study the properties of the discrete series constants, and show that they are completely characterized by these properties. Since the constants $c\left(w: E^{*}: \Phi^{+}\right)$depend only on data involving the Lie algebra $\mathfrak{z}$, we may as well assume that $G$ is acceptable and that $G=\Xi$, so that $A_{I}^{+}$is central in $G$. Now since the discrete series character transforms by the central character along its center, we may as well assume that $A_{I}^{+}=A_{I}^{0}=\{1\}$. That is, we assume that $G$ is a connected split semisimple Lie group and $A$ is a split Cartan subgroup. We may as well also assume that $G \subset G_{\mathbf{C}}$, where $G_{\mathbf{C}}$ is a simply connected complexification of $G$.

Under these assumptions, let $\Phi^{+}$be any choice of positive roots for $\Phi$ and $E^{*}$ any connected component of $E^{\prime}=E^{\prime}(\Phi)$. Then for any discrete series parameter $\lambda \in E^{*}$ and any $a \in A^{+}=\left\{a \in A^{0}: y \alpha(\log a)>0 \forall \alpha \in \Phi^{+}\right\}$, we have

$$
\Delta_{A}(a) \Theta_{\lambda}(a)=\sum_{w \in W(\Phi)} \operatorname{det} w c\left(w: E^{*}: \Phi^{+}\right) \exp \left((w \lambda)^{y} \log a\right) .
$$

We first show that the constants do not depend on the choice $A$ of split Cartan subgroup or the Cayley transform.

Lemma 2.1. Let $\mathfrak{h}$ be any split Cartan subalgebra of $\mathfrak{g}$ and let $c$ be any Cayley transform in $A d\left(\mathfrak{g}_{\mathbf{C}}\right)$ such that $c\left(\mathfrak{b}_{\mathbf{C}}\right)=\mathfrak{h}_{\mathbf{C}}$. Define $\Delta_{H}$ as above using $c \Phi^{+}$. Let $h \in H^{+}=\left\{h \in H^{0}: c \alpha(\log h)>0 \forall \alpha \in \Phi^{+}\right\}$. Then

$$
\Delta_{H}(h) \Theta_{\lambda}(h)=\sum_{w \in W(\Phi)} \operatorname{det} w c\left(w: E^{*}: \Phi^{+}\right) \exp \left((w \lambda)^{c} \log h\right) .
$$

Proof. Since $\mathfrak{h}$ and $\mathfrak{a}$ are both split Cartan subalgebras of $\mathfrak{g}$, there is $k \in K$ such that $k A k^{-1}=H$. Let $c_{1}=A d k \circ y: \mathfrak{b}_{\mathbf{C}} \rightarrow \mathfrak{h}_{\mathbf{C}}$. Then $c c_{1}^{-1}\left(\mathfrak{h}_{\mathbf{C}}\right)=\mathfrak{h}_{\mathbf{C}}$, and so represents an element of $W\left(\mathfrak{g}_{\mathbf{C}}, \mathfrak{h}_{\mathbf{C}}\right)$. But since $\mathfrak{h}$ is split, there is $k^{\prime} \in N_{K}(H)$ such that $c c_{1}^{-1}$ acts on $\mathfrak{h}_{\mathbf{C}}$ as $A d k^{\prime}$. Thus $c=A d k^{\prime} \circ c_{1}=A d\left(k^{\prime} k\right) \circ y$ as a mapping from $\mathfrak{b}_{\mathbf{C}}$ to $\mathfrak{h}_{\mathbf{C}}$, and $k^{\prime} k A\left(k^{\prime} k\right)^{-1}=k^{\prime} H\left(k^{\prime}\right)^{-1}=H$. Thus we can assume that $k$ is chosen so that $c=A d k \circ y$ as a mapping from $\mathfrak{b}_{\mathbf{C}}$ to $\mathfrak{h}_{\mathbf{C}}$. Then

$$
k A^{+} k^{-1}=\left\{h \in H^{0}: y \alpha\left(A d k^{-1} \log h\right)>0 \forall \alpha \in \Phi^{+}\right)=H^{+} .
$$


Fix $h \in H^{+}$and let $a=k^{-1} h k \in A^{+}$. Then

$$
\begin{gathered}
\Delta_{H}(h)=e^{c \rho}(h) \prod_{\alpha \in c \Phi^{+}}\left(1-e^{-\alpha}(h)\right)=e^{y \rho}\left(A d\left(k^{-1}\right) h\right) \prod_{\alpha \in y \Phi^{+}}\left(1-e^{-\alpha}\left(\operatorname{Ad}\left(k^{-1}\right) h\right)\right) \\
=e^{y \rho}(a) \prod_{\alpha \in y \Phi^{+}}\left(1-e^{-\alpha}(a)\right)=\Delta_{A}(a) .
\end{gathered}
$$

Now since $\Theta_{\lambda}(h)=\Theta_{\lambda}(a)$,

$$
\Delta_{H}(h) \Theta_{\lambda}(h)=\Delta_{A}(a) \Theta_{\lambda}(a)=\sum_{w \in W(\Phi)} \operatorname{det} w c\left(w: E^{*}: \Phi^{+}\right) \exp \left((w \lambda)^{y} \log a\right) .
$$

But $y^{-1} \log a=y^{-1} A d k^{-1} \log h=c^{-1} \log h$. Thus

$$
\exp \left((w \lambda)^{y} \log a\right)=\exp \left((w \lambda)^{c} \log h\right) .
$$

Lemma 2.2. Let $u \in W(\Phi)$. Then for all choices of $w \in W(\Phi), E^{*}$, and $\Phi^{+}$,

$$
c\left(u w: E^{*}: u \Phi^{+}\right)=c\left(w: E^{*}: \Phi^{+}\right) .
$$

Proof. Let $\lambda$ be a discrete series parameter in $E^{*}$ and let $\mathfrak{a}^{+}$be the positive Weyl chamber for $y \Phi^{+}$. Then for all $a \in A^{+}$,

$$
\Delta_{A}(a) \Theta_{\lambda}(a)=\sum_{w \in W(\Phi)} \operatorname{det} w c\left(w: E^{*}: \Phi^{+}\right) \exp \left((w \lambda)^{y} \log a\right) .
$$

Since $\mathfrak{a}$ is split, every element of $W(\Phi)^{y}=W(y \Phi)$ is induced on $\mathfrak{a}$ by an element of $G$. Thus there is $x \in G$ such that $x^{-1}=\exp \left(u^{y} \log a\right)$. Now $x^{-1} \in$ $\exp \left(u^{y} \mathfrak{a}^{+}\right)$, and $\Phi\left(u^{y} \mathfrak{a}^{+}\right)=u \Phi\left(\mathfrak{a}^{+}\right)=u \Phi^{+}$. Thus

$$
\begin{aligned}
& \Delta_{A}\left(x a x^{-1}\right) \Theta_{\lambda}\left(x a x^{-1}\right) \\
& \quad=\sum_{w \in W(\Phi)} \operatorname{det} w c\left(w: E^{*}: u \Phi^{+}\right) \exp \left((w \lambda)^{y}\left(u^{y} \log a\right)\right) .
\end{aligned}
$$

Using the change of variables, $w \mapsto u w$, we have

$$
\begin{aligned}
& \Delta_{A}\left(x a x^{-1}\right) \Theta_{\lambda}\left(x a x^{-1}\right) \\
& \quad=\sum_{w \in W(\Phi)} \operatorname{det} u \operatorname{det} w c\left(u w: E^{*}: u \Phi^{+}\right) \exp \left((u w \lambda)^{y}\left(u^{y} \log a\right)\right) \\
& \quad=\operatorname{det} u \sum_{w \in W(\Phi)} \operatorname{det} w c\left(u w: E^{*}: u \Phi^{+}\right) \exp \left((w \lambda)^{y} \log a\right) .
\end{aligned}
$$

But $\Theta_{\lambda}\left(x a x^{-1}\right)=\Theta_{\lambda}(a)$ and

$$
\Delta_{A}\left(x a x^{-1}\right)=\operatorname{det} u \Delta_{A}(a) .
$$

Thus we have

$$
\begin{aligned}
& \sum_{w \in W(\Phi)} \operatorname{det} w c\left(w: E^{*}: \Phi^{+}\right) \exp \left((w \lambda)^{y} \log a\right) \\
= & \sum_{w \in W(\Phi)} \operatorname{det} w c\left(u w: E^{*}: u \Phi^{+}\right) \exp \left((w \lambda)^{y} \log a\right) .
\end{aligned}
$$


Since the exponential terms are linearly independent, we must have

$$
c\left(u w: E^{*}: u \Phi^{+}\right)=c\left(w: E^{*}: \Phi^{+}\right)
$$

for all $w \in W(\Phi)$.

Remark. Lemma 2.2 is probably a consequence of [HC1, Corollary to Lemma 33]. However it is easier to reprove it than to track down all the notation.

Lemma 2.3. Let $t \in W\left(\Phi_{K}\right)$, where $\Phi_{K}$ denotes the set of compact roots in $\Phi$. Then for all choices of $w \in W(\Phi), E^{*}$, and $\Phi^{+}$,

$$
c\left(w t: t^{-1} E^{*}: \Phi^{+}\right)=c\left(w: E^{*}: \Phi^{+}\right) .
$$

Proof. Under the assumption that $G=\Xi$ we have $W\left(A_{I}^{+}\right)=W\left(\Phi_{K}\right)$ and

$$
c\left(w: E^{*}: \Phi\left(\mathfrak{a}^{+}\right)\right)=c_{H C}\left(w^{y}: E^{*}: A^{+}\right) .
$$

Thus the lemma follows from the equation

$$
c_{H C}\left(s u^{y}: u^{-1} E^{*}: A^{+}\right)=c_{H C}\left(s: E^{*}: A^{+}\right), u \in W\left(A_{I}^{+}\right),
$$

following Lemma 58 of [HC1].

Lemma 2.4. Suppose that there is $\tau \in E^{*}$ such that $\langle w \tau, \alpha\rangle>0$ for all $\alpha \in \Phi^{+}$. Then $c\left(w: E^{*}: \Phi^{+}\right)=0$.

Proof. Let $\mathfrak{a}^{+}$be the positive Weyl chamber for $y \Phi^{+}$. For each $\alpha \in \Phi$, let $H_{y \alpha} \in \mathfrak{a}$ correspond to $y \alpha$ by the usual Killing form identification of $\mathfrak{a}$ and $\mathfrak{a}^{*}$. Then $H=$ $\sum_{\alpha \in \Phi^{+}} H_{y \alpha} \in \mathfrak{a}^{+}$and $\left\langle(w \tau)^{y}, H\right\rangle=\left\langle\tau^{y},\left(w^{y}\right)^{-1} H\right\rangle>0$. Now by [HC1, Lemma 60], $c_{H C}\left(w^{y}: E^{*}: A^{+}\right)=0$. As in the proof of Lemma 2.3,

$$
c_{H C}\left(w^{y}: E^{*}: A^{+}\right)=c\left(w: E^{*}: \Phi^{+}\right) .
$$

Remark. Since $E^{*}$ is a connected component of $E^{\prime}(\Phi)$, the condition of Lemma 2.4 holds for some $\tau \in E^{*}$ if and only if it hold for all $\tau \in E^{*}$. Further, Lemma 2.4 is much weaker than [HC1, Lemma 60]. However, for the induction, we only need to know that the constants are zero in one chamber.

In order to completely characterize the constants $c\left(w: E^{*}: \Phi^{+}\right)$we will need a patching condition. As above we assume that $G$ is split and $A^{+} \subset A^{0}$, where $\mathfrak{a}$ is a split Cartan subalgebra. Fix $\alpha \in \Phi$ and let

$$
\Phi_{\alpha}=\{\beta \in \Phi:\langle\beta, \alpha\rangle=0\} .
$$

We will say that $\alpha$ is a good root of $\Phi$ if $\Phi_{\alpha}$ is spanned by strongly orthogonal noncompact roots.

Lemma 2.5. Let $\alpha \in \Phi$. Then $\alpha$ is a good root of $\Phi$ if and only if there is a $\theta$ stable Cartan subgroup $H=H_{I} H_{R}$ of $G$ with $H_{I}^{0} \subset B$ and $\Phi_{\alpha}=\Phi\left(\mathfrak{z}_{\mathbf{C}}, \mathfrak{b}_{\mathbf{C}}\right)$, where $\mathfrak{z}$ is the centralizer in $\mathfrak{g}$ of $H_{I}^{0}$.

Proof. Suppose that such a Cartan subgroup $H$ exists. Then $\Phi_{\alpha}$ is the root system of a Lie algebra $\mathfrak{z}$ (with respect to compact Cartan subalgebra $\mathfrak{b}$ ) which has a Cartan subalgebra $\mathfrak{h}$ which is split modulo the center of $\mathfrak{z}$. Thus $\Phi_{\alpha}$ is spanned by strongly orthogonal noncompact roots. 
Conversely, suppose that $\Phi_{\alpha}$ is spanned by the set $S$ of strongly orthogonal noncompact roots. Let $c_{S}=\prod_{\beta \in S} c_{\beta}$, where $c_{\beta}$ denotes the Cayley transform in $\beta$. Then $c_{S}\left(\mathfrak{b}_{\mathbf{C}}\right)=\mathfrak{h}_{\mathbf{C}}$, where $\mathfrak{h}=\mathfrak{h}_{I}+\mathfrak{h}_{R}$ is a Cartan subalgebra of $\mathfrak{g}$ with

$$
\mathfrak{h}_{I}=\mathfrak{h} \cap \mathfrak{k}=\{H \in \mathfrak{b}: \beta(H)=0 \forall \beta \in S\}=\mathbf{R} i H_{\alpha}
$$

and

$$
\mathfrak{h}_{R}=\mathfrak{h} \cap \mathfrak{p}=\sum_{\beta \in S} \mathbf{R} c_{S}\left(H_{\beta}\right),
$$

where for all $\beta \in \Phi, H_{\beta} \in \mathfrak{b}_{\mathbf{C}}$ corresponds to $\beta$ via the Killing form. Clearly $H_{I}^{0} \subset B$ and $\Phi_{\alpha}=\Phi\left(\mathfrak{z}_{\mathbf{C}}, \mathfrak{b}_{\mathbf{C}}\right)$.

Suppose that $\alpha$ is a good root of $\Phi$, and let $H$ be the Cartan subgroup with $H_{I}^{0} \subset B$ and $\Phi_{\alpha}=\Phi\left(\mathfrak{z}_{\mathbf{C}}, \mathfrak{b}_{\mathbf{C}}\right)$ given by Lemma 2.5 . Let $y^{\prime} \in A d\left(\mathfrak{z}_{\mathbf{C}}\right)$ be a Cayley transform with $y^{\prime} \mathfrak{b}_{\mathbf{C}}=\mathfrak{h}_{\mathbf{C}}$. Let $\Phi^{+}$be a choice of positive roots for which $\alpha$ is simple, and let $\Phi_{\alpha}^{+}=\Phi^{+} \cap \Phi_{\alpha}$,

$$
H_{R}^{+}=\left\{h_{2} \in H_{R}: y^{\prime} \beta\left(\log h_{2}\right)>0 \forall \beta \in \Phi_{\alpha}^{+}\right\} .
$$

Then if $\Delta_{H}$ is defined using $y^{\prime} \Phi^{+}$, for any $h_{1} \in H_{I}^{0}, h_{2} \in H_{R}^{+}$, by equation (2.6) we have

$$
\begin{gathered}
\Delta_{H}\left(h_{1} h_{2}\right) \Theta_{\lambda}\left(h_{1} h_{2}\right)=\sum_{t \in W \Xi \backslash W_{G}} \operatorname{det} t e^{t \lambda}\left(h_{1}\right) \\
\times \sum_{v \in W\left(\Phi_{\alpha}\right)} \operatorname{det} v c\left(v: t E^{*}: \Phi_{\alpha}^{+}\right) \exp \left((v t \lambda)^{y^{\prime}} \log h_{2}\right) .
\end{gathered}
$$

Thus when $\alpha$ is a good root we have associated discrete series constants $c\left(v: E^{*}: \Phi_{\alpha}^{+}\right), v \in W\left(\Phi_{\alpha}\right)$. In order to state the patching condition cleanly in our terminology, we will extend our definition. Let $w \in W(\Phi)$. Then we define $c\left(w: E^{*}: \Phi_{\alpha}^{+}\right)=0$ unless $w=v t, v \in W\left(\Phi_{\alpha}\right), t \in W\left(\Phi_{K}\right)$. When $w=v t, v \in$ $W\left(\Phi_{\alpha}\right), t \in W\left(\Phi_{K}\right)$, we define

$$
c\left(w: E^{*}: \Phi_{\alpha}^{+}\right)=c\left(v: t E^{*}: \Phi_{\alpha}^{+}\right) .
$$

Lemma 2.6. Suppose that $\alpha$ is a good root of $\Phi$ and is simple for the ordering $\Phi^{+}$. Then for all $w \in W(\Phi)$,

$$
c\left(w: E^{*}: \Phi^{+}\right)+c\left(s_{\alpha} w: E^{*}: \Phi^{+}\right)=c\left(w: E^{*}: \Phi_{\alpha}^{+}\right)+c\left(s_{\alpha} w: E^{*}: \Phi_{\alpha}^{+}\right) .
$$

Here $s_{\alpha}$ denotes the reflection corresponding to $\alpha$.

Proof. Such a patching condition for constants on the Lie algebra appears in [HC1, Lemma 26]. However it will be easier to derive our patching condition from the treatment by Knapp in $[\mathrm{K}, 13.5]$.

As in the proof of Lemma 2.5 , let $S$ be a collection of strongly orthogonal noncompact roots spanning $\Phi_{\alpha}$, and define $\mathfrak{h}_{\mathbf{C}}=c_{S}\left(\mathfrak{b}_{\mathbf{C}}\right)$. Then $c_{S} \Phi_{\alpha}$ is the set of real roots of $\mathfrak{h}$ in $\mathfrak{g}$, and $\pm c_{S} \alpha$ are the only roots which take pure imaginary values on $\mathfrak{h}$. Since $\mathfrak{g}$ is split, the root $c_{S} \alpha$ must be a noncompact imaginary root of $\mathfrak{h}$. (Otherwise $\mathfrak{h}$ would be maximally split in $\mathfrak{g}$.) Let $c_{1}$ be the Cayley transform in $c_{S} \alpha$. Then there is a split Cartan subalgebra $\mathfrak{a}^{\prime}$ of $\mathfrak{g}$ so that $\mathfrak{a}_{\mathbf{C}}{ }^{\prime}=c_{1} \mathfrak{h}_{\mathbf{C}}=c \mathfrak{b}_{\mathbf{C}}$, where $c=c_{1} c_{S}$. By Lemma 2.1, the constants occurring in the character formula 
for $\mathfrak{a}^{\prime}$ are the same as those occurring in the character formula for $\mathfrak{a}$. Thus we have the character formula

$$
\Delta_{A^{\prime}}\left(a^{\prime}\right) \Theta_{\lambda}\left(a^{\prime}\right)=\sum_{w \in W(\Phi)} \operatorname{det} w c\left(w: E^{*}: \Phi^{+}\right) \exp \left((w \lambda)^{c} \log a^{\prime}\right)
$$

for $a^{\prime} \in\left(A^{\prime}\right)^{+}=\left\{a^{\prime} \in A^{\prime}: c \alpha\left(\log a^{\prime}\right)>0 \forall \alpha \in \Phi^{+}\right\}$.

Compare this to $\left[\mathrm{K}\right.$, formula 13.30]. In $[\mathrm{K}, 13.30] \Theta_{\lambda}$ denotes the actual discrete series character, so (13.30) contains the term $(-1)^{q} \epsilon(\lambda)$. It contains constants $c_{K}\left(w, \lambda, \Delta_{L}^{+}\right), w \in W(\Phi)$, where $\Delta_{L}^{+}$is the positive system in $\Phi\left(\mathfrak{g}_{\mathbf{C}}, \mathfrak{a}_{\mathbf{C}}{ }^{\prime}\right)$ determined by $\left(A^{\prime}\right)^{+}$. Thus in our notation, $\Delta_{L}^{+}=c \Phi^{+}$. Thus for all $w \in W(\Phi)$ we have

$$
c\left(w: E^{*}: \Phi^{+}\right)=c_{K}\left(w, \lambda, c \Phi^{+}\right) .
$$

Now let $\alpha^{\prime}=c \alpha$. It is a simple root for $\Delta_{L}^{+}=c \Phi^{+}$. Knapp defines

$$
\Delta_{L, \alpha^{\prime}}^{+}=\left\{\beta \in \Delta_{L}^{+}:\left\langle\beta, \alpha^{\prime}\right\rangle=0\right\} .
$$

Thus $\Delta_{L, \alpha^{\prime}}^{+}=c \Phi_{\alpha}^{+}$, and the Cayley transform $d_{\alpha^{\prime}}$ used in $[\mathrm{K}]$ is just $c_{1}^{-1}$. Thus $d_{\alpha^{\prime}}\left(\mathfrak{a}_{\mathbf{C}}{ }^{\prime}\right)=\mathfrak{h}_{\mathbf{C}}$, and the Cayley transform $c^{*}: \mathfrak{b}_{\mathbf{C}} \rightarrow \mathfrak{h}_{\mathbf{C}}$ is $c^{*}=c_{1}^{-1} c=c_{S}$. Now $H_{I}^{0} \subset B$ is the connected component of $H_{I}$ containing $\left(A^{\prime}\right)_{I}^{0}=\{1\}$. As above, the constants $c\left(v: E^{*}: \Phi_{\alpha}^{+}\right), v \in W\left(\Phi_{\alpha}\right)$, are the constants occurring in the character formula

$$
\begin{gathered}
\Delta_{H}\left(h_{1} h_{2}\right) \Theta_{\lambda}\left(h_{1} h_{2}\right)=\sum_{t \in W \Xi \backslash W_{G}} \operatorname{det} t e^{t \lambda}\left(h_{1}\right) \\
\left.\times \sum_{v \in W\left(\Phi_{\alpha}\right)} \operatorname{det} v c\left(v: t E^{*}: \Phi_{\alpha}^{+}\right) \exp \left((v t \lambda)^{c^{*}} \log h_{2}\right)\right) .
\end{gathered}
$$

Note that $W\left(H_{I}^{0}\right)=W_{G} \cap W\left(\Phi_{\alpha}\right)$ is the set of all elements in $W_{G}$ which fix $\alpha$. Now since $W_{G}=W\left(\Phi_{K}\right)$ is generated by reflections in the compact roots, $W_{G} \cap W\left(\Phi_{\alpha}\right)$ is generated by reflections in compact roots which are orthogonal to $\alpha$. Thus $W\left(H_{I}^{0}\right)=W_{\Xi}=W\left(\Phi_{\alpha, K}\right)$, where $\Phi_{\alpha, K}=\Phi_{K} \cap \Phi_{\alpha}$. Thus the exponential terms occurring on the right hand side of the above equation are linearly independent.

The comparable formula from $[\mathrm{K}, 13.30]$ is

$$
\Delta_{H}\left(h_{1} h_{2}\right) \Theta_{\lambda}\left(h_{1} h_{2}\right)=\sum_{w \in W(\Phi)} \operatorname{det} w c_{K}\left(w, \lambda, \Delta_{L, \alpha^{\prime}}^{+}\right) e^{w \lambda}\left(h_{1}\right) \exp \left((w \lambda)^{c^{*}} \log h_{2}\right) .
$$

Comparing the coefficients of the different exponential terms, we see that $c_{K}\left(w, \lambda, \Delta_{L, \alpha^{\prime}}^{+}\right)=0$ unless there are $t \in W\left(\Phi_{K}\right), v \in W\left(\Phi_{\alpha}\right)$ such that $w=v t$. If $w=v t$ then

$$
c_{K}\left(w, \lambda, \Delta_{L, \alpha^{\prime}}^{+}\right)=c\left(v: t E^{*}: \Phi_{\alpha}^{+}\right) .
$$

Thus for all $w \in W(\Phi)$, using the extension of our constants to $W(\Phi)$, we have

$$
c_{K}\left(w, \lambda, \Delta_{L, \alpha^{\prime}}^{+}\right)=c\left(w: E^{*}: \Phi_{\alpha}^{+}\right) .
$$

The patching condition in $[\mathrm{K}$, formula 13.34$]$ is

$$
c\left(w, \lambda, \Delta_{L}^{+}\right)+c\left(s_{\alpha} w, \lambda, \Delta_{L}^{+}\right)=c\left(w, \lambda, \Delta_{L, \alpha^{\prime}}^{+}\right)+c\left(s_{\alpha} w, \lambda, \Delta_{L, \alpha^{\prime}}^{+}\right), \quad w \in W(\Phi) .
$$


In our notation the patching condition becomes

$$
c\left(w: E^{*}: \Phi^{+}\right)+c\left(s_{\alpha} w: E^{*}: \Phi^{+}\right)=c\left(w: E^{*}: \Phi_{\alpha}^{+}\right)+c\left(s_{\alpha} w: E^{*}: \Phi_{\alpha}^{+}\right) .
$$

The patching condition relates the constants for $\Phi$ to those of the lower rank root system $\Phi_{\alpha}$. We also need an initial condition. This comes from the discrete series character formula on the compact Cartan subgroup $B$, and corresponds to the case that $\Phi=\emptyset$. Then $E^{\prime}(\Phi)=E$ and $W(\Phi)=\{1\}$. It is consistent with our notation and equation (2.1) to define

$$
c(1: E: \emptyset)=c_{H C}(1: E: B)=1 .
$$

\section{Amplified Statement of the Main Theorem}

Let $\Phi$ be a root system spanned by strongly orthogonal noncompact roots, and $E$ a real vector space containing $\Phi$. Then for every connected component $E^{*}$ of $E^{\prime}(\Phi)$, every positive system $\Phi^{+}$for $\Phi$, and every $w \in W(\Phi)$, we have a constant $c\left(w: E^{*}: \Phi^{+}\right)$coming from discrete series character formulas as in $\S 2$. By Lemmas $2.2,2.3,2.4,2.6$, and equation (2.9), these constants satisfy the following conditions.

For all $u, w \in W, v \in W_{K}=W\left(\Phi_{K}\right)$,

$$
\begin{gathered}
c\left(u w: E^{*}: u \Phi^{+}\right)=c\left(w: E^{*}: \Phi^{+}\right) \\
c\left(w v: v^{-1} E^{*}: \Phi^{+}\right)=c\left(w: E^{*}: \Phi^{+}\right)
\end{gathered}
$$

Let $\alpha$ be a good root of $\Phi$ which is simple for $\Phi^{+}$. Then, using the notation of Lemma 2.6,

$$
c\left(w: E^{*}: \Phi^{+}\right)+c\left(s_{\alpha} w: E^{*}: \Phi^{+}\right)=c\left(w: E^{*}: \Phi_{\alpha}^{+}\right)+c\left(s_{\alpha} w: E^{*}: \Phi_{\alpha}^{+}\right) .
$$

Since $\Phi$ is spanned by orthogonal roots, every irreducible factor of $\Phi$ will be of type $A_{1}, B_{n}, C_{n}, D_{2 n}, E_{7}, E_{8}, F_{4}$, or $G_{2}$.

Definition. If $\Phi$ is irreducible of type $A_{1}, B_{2 n+1}, D_{2 n}, E_{7}, E_{8}$, or $G_{2}$, we will say that $\Phi^{+}$is a good choice of positive roots if all simple roots are noncompact. If $\Phi$ is of type $B_{2 n}, C_{n}$, or $F_{4}$, we will say that $\Phi^{+}$is a good choice of positive roots if all long simple roots are noncompact and all short simple roots are compact.

Lemma 3.1. Let $\Phi$ be a root system spanned by strongly orthogonal noncompact roots. Then a good choice of positive roots exists. Further, if $\alpha$ is any simple root for a good choice of positive roots, then $\alpha$ is a good root.

It is enough to prove Lemma 3.1 in the case that $\Phi$ is irreducible. It is checked for each case in $\S 5$.

Lemma 3.2. Properties (3.1)-(3.5) determine the constants $c\left(w: E^{*}: \Phi^{+}\right)$completely. 
Proof. The constants are defined by (3.4) if rank $\Phi=0$. Let $\Phi$ have rank $n$ and assume inductively that the constants are completely defined for root systems of rank less than $n$. Let $\Phi_{g}^{+}$be a good choice of positive roots. Then any choice $\Phi^{+}$is of the form $\Phi^{+}=u \Phi_{g}^{+}$for some $u \in W$. Now for any $w \in W, E^{*} \subset E^{\prime}$, using (3.1),

$$
c\left(w: E^{*}: \Phi^{+}\right)=c\left(u^{-1} w: E^{*}: \Phi_{g}^{+}\right) .
$$

Thus it is enough to define the constants for $\Phi_{g}^{+}$. For $\Phi_{g}^{+}$we have patching conditions for every simple root, so as usual we can use (3.3), (3.5), and the induction hypothesis applied to the root systems $\Phi_{\alpha}^{+}$to determine all the constants corresponding to $\Phi_{g}^{+}$.

Suppose $\Phi_{1}$ and $\Phi_{2}$ are two root systems as above in real vector spaces $E_{1}$ and $E_{2}$ with positive roots $\Phi_{i}^{+}$and compact roots $\Phi_{i, K}, i=1,2$. Let $\psi: E_{1} \rightarrow E_{2}$ be an isomorphism such that

$$
\psi\left(\Phi_{1}^{+}\right)=\Phi_{2}^{+}, \quad \psi\left(\Phi_{1, K}\right)=\Phi_{2, K} .
$$

For each $E_{1}^{*} \subset E_{1}^{\prime}$, we have $E_{2}^{*}=\psi\left(E_{1}^{*}\right) \subset E_{2}^{\prime}$. Also, for any $w_{1} \in W\left(\Phi_{1}\right), w_{2}=$ $\psi w_{1} \psi^{-1} \in W\left(\Phi_{2}\right)$. Now because $\psi$ preserves all the structure used to characterize the constants, for all $w_{1} \in W\left(\Phi_{1}\right), E_{1}^{*} \subset E_{1}^{\prime}$ we have

$$
c\left(w_{2}: E_{2}^{*}: \Phi_{2}^{+}\right)=c\left(\psi w_{1} \psi^{-1}: \psi E_{1}^{*}: \psi \Phi_{1}^{+}\right)=c\left(w_{1}: E_{1}^{*}: \Phi_{1}^{+}\right) .
$$

Let $\Phi$ be a root system spanned by strongly orthogonal noncompact roots as above, and write $W=W(\Phi)$. Recall that a root system $\phi \subset \Phi$ is called a twostructure for $\Phi$ if:

(i) all irreducible factors of $\phi$ are of type $A_{1}$ or $B_{2} \simeq C_{2}$;

(ii) for any choice $\phi^{+}$of positive roots for $\phi,\left\{w \in W: w \phi^{+}=\phi^{+}\right\}$contains no elements of determinant -1 . Property (ii) forces a two-structure $\phi$ to be as big as possible; in particular rank $\phi=\operatorname{rank} \Phi$.

Let $\mathcal{T}(\Phi)$ denote the set of all two-structures of $\Phi$ and let $\Phi^{+}$be a choice of positive roots for $\Phi$. For each $\phi \in \mathcal{T}(\Phi)$, let $\phi^{+}=\phi \cap \Phi^{+}$. Define

$$
W\left(\phi, \Phi^{+}\right)=\left\{\sigma \in W: \sigma \phi^{+} \subset \Phi^{+}\right\} .
$$

Then as in [H3] or [K, p. 501] we can associate to each $\phi \in \mathcal{T}(\Phi)$ a sign $\epsilon\left(\phi: \Phi^{+}\right)=$ \pm 1 . These signs have the property that for any $\phi \in \mathcal{T}(\Phi), \sigma \in W\left(\phi, \Phi^{+}\right), w \in W$,

$$
\begin{gathered}
\epsilon\left(\sigma \phi: \Phi^{+}\right)=\operatorname{det} \sigma \epsilon\left(\phi: \Phi^{+}\right) ; \\
\epsilon\left(w \phi: w \Phi^{+}\right)=\epsilon\left(\phi: \Phi^{+}\right) .
\end{gathered}
$$

They satisfy the following compatibility condition with patching. Let $\alpha \in \Phi$ and $\phi \in \mathcal{T}(\Phi)$. Then if $\alpha \in \phi$, we define $\phi_{\alpha}=\phi \cap \Phi_{\alpha}$.

Lemma 3.3. Let $\alpha$ be any root of $\Phi$ and let $\Phi^{+}$be a choice of positive roots for which $\alpha$ is simple. Suppose $\phi \in \mathcal{T}(\Phi)$ is such that $\alpha \in \phi$ and $\phi_{\alpha} \in \mathcal{T}\left(\Phi_{\alpha}\right)$. Then

$$
\epsilon\left(\phi: \Phi^{+}\right)=\epsilon\left(\phi_{\alpha}: \Phi_{\alpha}^{+}\right) .
$$

Lemma 3.3 was stated without proof in [H3]. A proof is given in $\S 5$.

Two structures were used in [H1], [H3] to obtain a formula for the constants occurring in averaged discrete series character formulas. For $E^{*} \subset E^{\prime}$, define the averaged discrete series constant

$$
\bar{c}\left(E^{*}: \Phi^{+}\right)=\left[W_{K}\right]^{-1} \sum_{w \in W} c\left(w: w^{-1} E^{*}: \Phi^{+}\right) .
$$


Averaged discrete series constants corresponding to $\phi \in \mathcal{T}(\Phi)$ can be defined as follows. Let $\phi \in \mathcal{T}(\Phi)$. All irreducible factors of $\phi$ are of type $A_{1}$ or $B_{2} \simeq C_{2}$. By identifying irreducible factors of type $A_{1}$ with the root system of the Lie algebra of $S L(2, \mathbf{R})$ and irreducible factors of type $B_{2} \simeq C_{2}$ with the root system of the Lie algebra of $S p(2, \mathbf{R})$, we obtain a subset $\phi_{K}$ of compact roots. Every root occurring in an $A_{1}$ factor will be noncompact, and any long root occurring in a $B_{2} \simeq C_{2}$ factor will be noncompact. In $B_{2} \simeq C_{2}$ factors, one short root (and its negative) will be compact, the other will be noncompact. Which short root is compact will depend on the identification used. Fix a choice of $\phi_{K}$ as above. Then for any choice of positive roots $\phi^{+}, w \in W(\phi)$, and $E_{\phi}^{*} \subset E(\phi)^{\prime}$ we have constants $c\left(w: E_{\phi}^{*}: \phi^{+}\right)$. Now let $E^{*}$ be a connected component of $E^{\prime}=E^{\prime}(\Phi)$. There is a unique connected component $E_{\phi}^{*}$ of $E^{\prime}(\phi)$ which contains it, and we write

$$
c\left(w: E^{*}: \phi^{+}\right)=c\left(w: E_{\phi}^{*}: \phi^{+}\right) .
$$

Define

$$
\bar{c}\left(E^{*}: \phi^{+}\right)=\left[W\left(\phi_{K}\right)\right]^{-1} \sum_{w \in W(\phi)} c\left(w: w^{-1} E^{*}: \phi^{+}\right) .
$$

Although the constants $c\left(w: E^{*}: \phi^{+}\right)$depend on the identifications used to define $\phi_{K}$, the averaged constants $\bar{c}\left(E^{*}: \phi^{+}\right)$are independent of the identifications. It is proved in [H1], [H3] that

$$
\bar{c}\left(E^{*}: \Phi^{+}\right)=\sum_{\phi \in \mathcal{T}(\Phi)} \epsilon\left(\phi: \Phi^{+}\right) \bar{c}\left(E^{*}: \phi^{+}\right),
$$

where $\phi^{+}=\phi \cap \Phi^{+}$for all $\phi \in \mathcal{T}(\Phi)$.

We want to obtain a formula similar to (3.9) for the (unaveraged) constants $c\left(w: E^{*}: \Phi^{+}\right)$. We will say a two-structure for $\Phi$ is of noncompact type if $\phi$ is spanned by a collection of strongly orthogonal noncompact roots of $\Phi$. This is equivalent to the condition that all long roots of $\phi$ are noncompact in $\Phi$, where by a long root of $\phi$ we mean any root in an $A_{1}$ factor and any long root in a $B_{2} \simeq C_{2}$ factor. Write $\mathcal{T}_{n}(\Phi)$ for the two-structures of noncompact type for $\Phi$. Then for any $\phi \in \mathcal{T}_{n}(\Phi), \phi_{K}=\phi \cap \Phi_{K}$ gives a canonical choice for compact roots in $\phi$. For $\phi \in \mathcal{T}_{n}(\Phi)$, we will always assume that this choice is used in defining discrete series constants $c\left(w: E^{*}: \phi^{+}\right), w \in W(\phi), E^{*} \subset E^{\prime}$.

To get a direct analogue of formula (3.9) for the unaveraged constants, we would like to express the constants $c\left(w: E^{*}: \Phi^{+}\right), w \in W$, in terms of $c\left(w: E^{*}: \phi^{+}\right)$, $\phi \in \mathcal{T}_{n}(\Phi)$. However, $W=W(\Phi)$ is not a subset of $W(\phi)$. Thus $c\left(w: E^{*}: \phi^{+}\right)$is not defined for arbitrary $w \in W$. We can get around this as follows. Recall from (3.1) that for any $w \in W$ and any choice of $\Phi^{+}$and $E^{*}$,

$$
c\left(w: E^{*}: \Phi^{+}\right)=c\left(1: E^{*}: w^{-1} \Phi^{+}\right) .
$$

Thus it is enough to have formulas for the constants

$$
d\left(E^{*}: \Phi^{+}\right)=c\left(1: E^{*}: \Phi^{+}\right)
$$

for any choice of $\Phi^{+}$and $E^{*}$. We also have constants $d\left(E^{*}: \phi^{+}\right)=c\left(1: E^{*}: \phi^{+}\right)$ for every $\phi \in \mathcal{T}_{n}(\Phi)$.

We will prove the following theorem in the next section. It contains a constant $c(\Phi)$ which does not occur in (3.9) and is defined as follows. For any $\phi \in \mathcal{T}(\Phi)$ let $L=L(\Phi) \subset L(\phi)$, where $L(\Phi), L(\phi)$ denote the weight lattices of $\Phi$ and $\phi$ respectively. Let $c(\Phi)=[L(\phi): L(\Phi)]$. It is independent of the choice of $\phi$, since 
all $\phi \in \mathcal{T}(\Phi)$ are conjugate via $W=W(\Phi)$. Its values for $\Phi$ irreducible are given by the following table, which also gives the type of $\phi$ in each case.

$$
\begin{array}{lllllllllll}
\Phi & A_{1} & B_{2 n} & B_{2 n+1} & C_{2 n} & C_{2 n+1} & D_{2 n} & E_{7} & E_{8} & F_{4} & G_{2} \\
\phi & A_{1} & B_{2}^{n} & B_{2}^{n} \times B_{1} & C_{2}^{n} & C_{2}^{n} \times C_{1} & A_{1}^{2 n} & A_{1}^{7} & A_{1}^{8} & B_{2}^{2} & A_{1}^{2} \\
c(\Phi) & 1 & 2^{n-1} & 2^{n} & 1 & 1 & 2^{n-1} & 2^{3} & 2^{4} & 2 & 2
\end{array}
$$

Theorem 3.4. Let $E^{*}$ be any connected component of $E^{\prime}$ and let $\Phi^{+}$be any choice of positive roots for $\Phi$. For all $\phi \in \mathcal{T}_{n}(\Phi)$, let $\phi^{+}=\phi \cap \Phi^{+}$. Then

$$
d\left(E^{*}: \Phi^{+}\right)=c(\Phi) \sum_{\phi \in \mathcal{T}_{n}(\Phi)} \epsilon\left(\phi: \Phi^{+}\right) d\left(E^{*}: \phi^{+}\right) .
$$

Equivalently, for any $w \in W(\Phi)$ and $E^{*}, \Phi^{+}$as above,

$$
c\left(w: E^{*}: \Phi^{+}\right)=c(\Phi) \sum_{\phi \in \mathcal{T}_{n}(\Phi)} \epsilon\left(\phi: w^{-1} \Phi^{+}\right) c\left(1: E^{*}: \phi \cap w^{-1} \Phi^{+}\right) .
$$

\section{Proof of Theorem 3.4}

Let $\Phi$ be a root system spanned by strongly orthogonal noncompact roots as in $\S 3$. For any choice of positive roots $\Phi^{+}$and $w \in W, E^{*} \subset E^{\prime}$, define

$$
s\left(w: E^{*}: \Phi^{+}\right)=c(\Phi) \sum_{\phi \in \mathcal{T}_{n}(\Phi)} \epsilon\left(\phi: w^{-1} \Phi^{+}\right) c\left(1: E^{*}: \phi \cap w^{-1} \Phi^{+}\right) .
$$

In order to prove Theorem 3.4 we must show that $c\left(w: E^{*}: \Phi^{+}\right)=s\left(w: E^{*}: \Phi^{+}\right)$. We will do this by showing that the constants $s\left(w: E^{*}: \Phi^{+}\right)$satisfy the conditions (3.1)-(3.5) characterizing the discrete series constants. Checking the first four of these is easy.

Lemma 4.1. The constants $s\left(w: E^{*}: \Phi^{+}\right)$satisfy conditions (3.1)-(3.4).

Proof. Let $u \in W$. Then

$$
\begin{aligned}
& s\left(u w: E^{*}: u \Phi^{+}\right) \\
& \quad=c(\Phi) \sum_{\phi \in \mathcal{T}_{n}(\Phi)} \epsilon\left(\phi: w^{-1} u^{-1} u \Phi^{+}\right) c\left(1: E^{*}: \phi \cap w^{-1} u^{-1} u \Phi^{+}\right)=s\left(w: E^{*}: \Phi^{+}\right) .
\end{aligned}
$$

Thus the constants $s\left(w: E^{*}: \Phi^{+}\right)$satisfy (3.1).

Let $v \in W_{K}=W\left(\Phi_{K}\right)$. Then $\alpha \mapsto v^{-1} \alpha$ preserves compact and noncompact roots of $\Phi$. Thus for any $\phi \in \mathcal{T}_{n}(\Phi), v^{-1} \phi \in \mathcal{T}_{n}(\Phi)$ also. Define the mapping $\psi_{v}: E \rightarrow E$ by $\psi_{v}(\tau)=v^{-1} \tau, \tau \in E$. Then $\psi_{v}(\phi)=v^{-1} \phi$. Further,

$$
\psi_{v}\left(\phi_{K}\right)=v^{-1}\left(\phi \cap \Phi_{K}\right)=v^{-1} \phi \cap \Phi_{K}=\left(v^{-1} \phi\right)_{K} .
$$

Thus, using (3.6),

$$
\begin{aligned}
c\left(1: v^{-1} E^{*}: v^{-1} \phi \cap v^{-1} w^{-1} \Phi^{+}\right) & =c\left(1: v^{-1} E^{*}: v^{-1}\left(\phi \cap w^{-1} \Phi^{+}\right)\right) \\
& =c\left(1: E^{*}: \phi \cap w^{-1} \Phi^{+}\right) .
\end{aligned}
$$


Now using the change of variables $\phi \mapsto v^{-1} \phi$ and (3.8), we have

$$
\begin{aligned}
s(w v & \left.: v^{-1} E^{*}: \Phi^{+}\right) \\
& =c(\Phi) \sum_{\phi \in \mathcal{T}_{n}(\Phi)} \epsilon\left(\phi: v^{-1} w^{-1} \Phi^{+}\right) c\left(1: v^{-1} E^{*}: \phi \cap v^{-1} w^{-1} \Phi^{+}\right) \\
& =c(\Phi) \sum_{\phi \in \mathcal{T}_{n}(\Phi)} \epsilon\left(v^{-1} \phi: v^{-1} w^{-1} \Phi^{+}\right) c\left(1: v^{-1} E^{*}: v^{-1} \phi \cap v^{-1} w^{-1} \Phi^{+}\right) \\
& =s\left(w: E^{*}: \Phi^{+}\right) .
\end{aligned}
$$

Thus the constants $s\left(w: E^{*}: \Phi^{+}\right)$satisfy (3.2).

Suppose that there is $\tau \in E^{*}$ such that $\langle w \tau, \alpha\rangle>0$ for all $\alpha \in \Phi^{+}$. Then for any $\phi \in \mathcal{T}_{n}(\Phi),\langle\tau, \beta\rangle>0$ for all $\beta \in \phi \cap w^{-1} \Phi^{+}$, so that $c\left(1: E^{*}: \phi \cap w^{-1} \Phi^{+}\right)=0$. Thus $s\left(w: E^{*}: \Phi^{+}\right)=0$, and the constants satisfy (3.3).

Finally, when $\Phi=\emptyset$, we have $\mathcal{T}_{n}(\Phi)=\{\emptyset\}, c(\Phi)=1, \epsilon(\emptyset: \emptyset)=1$, and $c(1: E: \emptyset)=1$. Thus $s(1: E: \emptyset)=1$ and the constants satisfy (3.4).

It remains to show that the constants $s\left(w: E^{*}: \Phi^{+}\right)$satisfy the patching condition (3.5). Thus for the remainder of this section we assume that $\Phi^{+}$is a good choice of positive roots and fix a simple root $\alpha$ for $\Phi^{+}$. If $w_{1} \in W\left(\Phi_{\alpha}\right), w_{2} \in W_{K}$, define

$$
s\left(w_{1} w_{2}: E^{*}: \Phi_{\alpha}^{+}\right)=s\left(w_{1}:\left(w_{2} E^{*}\right)_{\alpha}: \Phi_{\alpha}^{+}\right) .
$$

Define $s\left(w: E^{*}: \Phi_{\alpha}^{+}\right)=0$ if $w \notin W\left(\Phi_{\alpha}\right) W_{K}$. We must show that

$$
s\left(w: E^{*}: \Phi^{+}\right)+s\left(s_{\alpha} w: E^{*}: \Phi^{+}\right)=s\left(w: E^{*}: \Phi_{\alpha}^{+}\right)+s\left(s_{\alpha} w: E^{*}: \Phi_{\alpha}^{+}\right) .
$$

We will first simplify the left hand side of equation (4.1). Define

$$
\mathcal{T}_{n}\left(\Phi, w^{-1} \alpha\right)=\left\{\phi \in \mathcal{T}_{n}(\Phi): w^{-1} \alpha \in \phi\right\} .
$$

Lemma 4.2.

$$
\begin{gathered}
s\left(w: E^{*}: \Phi^{+}\right)+s\left(s_{\alpha} w: E^{*}: \Phi^{+}\right) \\
=c(\Phi) \sum_{\phi \in \mathcal{T}_{n}\left(\Phi, w^{-1} \alpha\right)} \epsilon\left(\phi: w^{-1} \Phi^{+}\right)\left[c\left(1: E^{*}: \phi \cap w^{-1} \Phi^{+}\right)\right. \\
\left.+c\left(1: E^{*}: \phi \cap w^{-1} s_{\alpha} \Phi^{+}\right)\right]
\end{gathered}
$$

Proof. By definition we have

$$
\begin{aligned}
& s\left(w: E^{*}: \Phi^{+}\right)+s\left(s_{\alpha} w: E^{*}: \Phi^{+}\right) \\
& =c(\Phi) \sum_{\phi \in \mathcal{T}_{n}(\Phi)}\left[\epsilon\left(\phi: w^{-1} \Phi^{+}\right) c\left(1: E^{*}: \phi \cap w^{-1} \Phi^{+}\right)\right. \\
& \left.\quad+\epsilon\left(\phi: w^{-1} s_{\alpha} \Phi^{+}\right) c\left(1: E^{*}: \phi \cap w^{-1} s_{\alpha} \Phi^{+}\right)\right] .
\end{aligned}
$$

By (3.8), $\epsilon\left(\phi: w^{-1} \Phi^{+}\right)=\epsilon\left(w \phi: \Phi^{+}\right)$and $\epsilon\left(\phi: w^{-1} s_{\alpha} \Phi^{+}\right)=\epsilon\left(s_{\alpha} w \phi: \Phi^{+}\right)$.

Suppose first that $\phi \notin \mathcal{T}_{n}\left(\Phi, w^{-1} \alpha\right)$. Then $\alpha \notin w \phi$. Since $\alpha$ is simple for $\Phi^{+}$, this implies that

$$
w \phi \cap s_{\alpha} \Phi^{+}=w \phi \cap \Phi^{+} .
$$

Thus $s_{\alpha}\left(w \phi \cap \Phi^{+}\right) \subset \Phi^{+}$and $s_{\alpha} \in W\left(w \phi, \Phi^{+}\right)$. Using (3.7),

$$
\epsilon\left(s_{\alpha} w \phi: \Phi^{+}\right)=-\epsilon\left(w \phi: \Phi^{+}\right) .
$$

Further,

$$
\phi \cap w^{-1} s_{\alpha} \Phi^{+}=w^{-1}\left(w \phi \cap s_{\alpha} \Phi^{+}\right)=w^{-1}\left(w \phi \cap \Phi^{+}\right)=\phi \cap w^{-1} \Phi^{+},
$$


so that

$$
c\left(1: E^{*}: \phi \cap w^{-1} s_{\alpha} \Phi^{+}\right)=c\left(1: E^{*}: \phi \cap w^{-1} \Phi^{+}\right) .
$$

Thus in this case

$$
\begin{aligned}
\epsilon(\phi & \left.: w^{-1} \Phi^{+}\right) c\left(1: E^{*}: \phi \cap w^{-1} \Phi^{+}\right) \\
& +\epsilon\left(\phi: w^{-1} s_{\alpha} \Phi^{+}\right) c\left(1: E^{*}: \phi \cap w^{-1} s_{\alpha} \Phi^{+}\right)=0 .
\end{aligned}
$$

Now if $\phi \in \mathcal{T}_{n}\left(\Phi, w^{-1} \alpha\right)$, then $\alpha \in w \phi$, so that $s_{\alpha} w \phi=w \phi$ and

$$
\epsilon\left(w \phi: \Phi^{+}\right)=\epsilon\left(s_{\alpha} w \phi: \Phi^{+}\right) .
$$

Let $\phi \in \mathcal{T}_{n}(\Phi)$. Recall that a root $\beta$ of $\phi$ is called a long root of $\phi$ if it is in an irreducible factor of $\phi$ of type $A_{1}$ or is a long root in an irreducible factor of type $B_{2} \simeq C_{2}$. It is called a short root of $\phi$ if it is a short root in an irreducible factor of type $B_{2} \simeq C_{2}$.

Lemma 4.3. Let $\phi \in \mathcal{T}_{n}(\Phi)$ and let $\beta \in \phi$. Then $\beta$ is a good root of $\phi$ unless $\beta$ is a short root of $\phi$ and is noncompact. Let $\phi^{+}$be a choice of positive roots for $\phi$ for which $\beta$ is simple. Then for all $E^{*} \subset E^{\prime}$,

$$
c\left(1: E^{*}: \phi^{+}\right)+c\left(s_{\beta}: E^{*}: \phi^{+}\right)=0
$$

if $\beta$ is not a good root of $\phi$. If $\beta$ is a good root of $\phi$, then

$$
c\left(1: E^{*}: \phi^{+}\right)+c\left(s_{\beta}: E^{*}: \phi^{+}\right)=c(\beta, \phi) c\left(1: E^{*}: \phi_{\beta}^{+}\right),
$$

where

$$
c(\beta, \phi)= \begin{cases}1, & \text { if } \beta \text { is a long root of } \phi ; \\ 2, & \text { if } \beta \text { is short root of } \phi .\end{cases}
$$

Proof. Write $\phi=\phi^{\prime \prime} \cup \phi^{\prime}$, where $\phi^{\prime}$ is the irreducible factor of $\phi$ containing $\beta$.

If $\phi^{\prime}$ is of type $A_{1}$, then $\phi_{\beta}=\phi^{\prime \prime}$ is spanned by strongly orthogonal noncompact roots, so $\beta$ is a good root of $\phi$. By (3.5) we have

$$
c\left(1: E^{*}: \phi^{+}\right)+c\left(s_{\beta}: E^{*}: \phi^{+}\right)=c\left(1: E^{*}: \phi_{\beta}^{+}\right)+c\left(s_{\beta}: E^{*}: \phi_{\beta}^{+}\right) .
$$

But $W\left(\phi_{\beta}\right) W_{K}(\phi)=W\left(\phi^{\prime \prime}\right)$, since $W_{K}(\phi) \subset W\left(\phi^{\prime \prime}\right)$. Thus $s_{\beta} \notin W\left(\phi_{\beta}\right) W_{K}(\phi)$, and so $c\left(s_{\beta}: E^{*}: \phi_{\beta}^{+}\right)=0$.

Suppose that $\phi^{\prime}$ is of type $B_{2} \simeq C_{2}$. We will use the notation

$$
\phi^{\prime}=\left\{ \pm e_{1} \pm e_{2}, \pm 2 e_{1}, \pm 2 e_{2}\right\}
$$

where the short root $e_{1}-e_{2}$ is compact and the roots $2 e_{1}, 2 e_{2}, e_{1}+e_{2}$ are noncompact. Then $\phi_{e_{1}+e_{2}}=\phi^{\prime \prime} \cup\left\{ \pm\left(e_{1}-e_{2}\right)\right\}$ is not spanned by noncompact roots, so $e_{1}+e_{2}$ is not a good root of $\phi$. However $\phi_{e_{1}-e_{2}}=\phi^{\prime \prime} \cup\left\{ \pm\left(e_{1}+e_{2}\right)\right\}$ and $\phi_{2 e_{i}}=\phi^{\prime \prime} \cup\left\{ \pm 2 e_{j}\right\}$ for $i, j=1,2, i \neq j$, so that $e_{1}-e_{2}, 2 e_{1}$, and $2 e_{2}$ are good roots of $\phi$.

Let $\beta=e_{1}+e_{2}$, and let $\phi^{+}$be a positive system for which $\beta$ is simple. Let $u=s_{2 e_{2}}$. Then $\alpha=u \beta=e_{1}-e_{2}$ is a good root and is simple for the positive system $u \phi^{+}$. Now since $u s_{\beta}=s_{\alpha} u$,

$$
\begin{gathered}
c\left(1: E^{*}: \phi^{+}\right)+c\left(s_{\beta}: E^{*}: \phi^{+}\right)=c\left(u: E^{*}: u \phi^{+}\right)+c\left(u s_{\beta}: E^{*}: u \phi^{+}\right) \\
=c\left(u: E^{*}: u \phi^{+}\right)+c\left(s_{\alpha} u: E^{*}: u \phi^{+}\right)
\end{gathered}
$$




$$
=c\left(u: E^{*}: \phi_{\alpha} \cap u \phi^{+}\right)+c\left(s_{\alpha} u: E^{*}: \phi_{\alpha} \cap u \phi^{+}\right) .
$$

But $\phi_{\alpha}=\phi^{\prime \prime} \cup\{ \pm \beta\}$. Thus $W\left(\phi_{\alpha}\right) W_{K}(\phi)$ is generated by $W\left(\phi^{\prime \prime}\right), s_{\alpha}$ and $s_{\beta}$. In particular, $v \beta= \pm \beta$ for all $v \in W\left(\phi_{\alpha}\right) W_{K}(\phi)$. But $u \beta=\alpha$ and $s_{\alpha} u \beta=-\alpha$. Thus $u$ and $s_{\alpha} u$ are both not elements of $W\left(\phi_{\alpha}\right) W_{K}(\phi)$, and we have

$$
c\left(u: E^{*}: \phi_{\alpha} \cap u \phi^{+}\right)=c\left(s_{\alpha} u: E^{*}: \phi_{\alpha} \cap u \phi^{+}\right)=0
$$

by definition.

Now suppose that $\beta$ is one of the good roots, and let $\phi^{+}$be an ordering for which $\beta$ is simple. Then we have the patching condition

$$
c\left(1: E^{*}: \phi^{+}\right)+c\left(s_{\beta}: E^{*}: \phi^{+}\right)=c\left(1: E^{*}: \phi_{\beta}^{+}\right)+c\left(s_{\beta}: E^{*}: \phi_{\beta}^{+}\right) .
$$

If $\beta=e_{1}-e_{2}$ is the short compact root, then $s_{\beta} \in W_{K}(\phi)$, so

$$
c\left(s_{\beta}: E^{*}: \phi_{\beta}^{+}\right)=c\left(1:\left(s_{\beta} E^{*}\right)_{\beta}: \phi_{\beta}^{+}\right)=c\left(1: E_{\beta}^{*}: \phi_{\beta}^{+}\right)=c\left(1: E^{*}: \phi_{\beta}^{+}\right) .
$$

If $\beta$ is one of the long roots, then $s_{\beta} \notin W\left(\phi_{\beta}\right) W_{K}(\phi)$, so that $c\left(s_{\beta}: E^{*}: \phi_{\beta}^{+}\right)=$ 0 .

Let $\beta_{1}, \beta_{2}$ be any roots of $\Phi$. We say $\beta_{1} \equiv \beta_{2}$ if either both $\beta_{1}$ and $\beta_{2}$ are compact or both are noncompact.

Lemma 4.4. Suppose that the left hand side of (4.1) is non-zero. Then $w^{-1} \alpha \equiv \alpha$.

Proof. By Lemma 4.2, the left hand side of (4.1) is

$$
c(\Phi) \sum_{\phi \in \mathcal{T}_{n}\left(\Phi, w^{-1} \alpha\right)} \epsilon\left(\phi: w^{-1} \Phi^{+}\right)\left[c\left(1: E^{*}: \phi \cap w^{-1} \Phi^{+}\right)+c\left(1: E^{*}: \phi \cap w^{-1} s_{\alpha} \Phi^{+}\right)\right] .
$$

Suppose that $\alpha$ is any simple root in a factor of type $A_{1}, D_{2 n}, E_{7}, E_{8}$, or $G_{2}$, or is a long simple root in a factor of type $B_{n}, C_{n}$, or $F_{4}$. Then $\alpha$ is noncompact since $\Phi^{+}$is a good choice of positive roots. If the left hand side of (4.1) is non-zero, then $w^{-1} \alpha \in \phi$ for at least one $\phi \in \mathcal{T}_{n}(\Phi)$. But $w^{-1} \alpha$ is in a factor of type $A_{1}$ of $\phi$ or is a long root in a factor of type $B_{2} \simeq C_{2}$, and hence is noncompact.

Thus $w^{-1} \alpha \equiv \alpha$.

Now suppose that $\alpha$ is a short simple root in a factor of type $B_{2 n}, C_{n}$, or $F_{4}$. Then $\alpha$ must be compact. Suppose that $\beta=w^{-1} \alpha$ is noncompact. Fix $\phi \in \mathcal{T}_{n}\left(\Phi, w^{-1} \alpha\right)$. Then $\beta \in \phi$ is a short noncompact root which is simple for the ordering $\phi \cap w^{-1} \Phi^{+}$. Furthermore, $w^{-1} s_{\alpha}=s_{\beta} w^{-1}$, so that

$$
c\left(1: E^{*}: \phi \cap w^{-1} s_{\alpha} \Phi^{+}\right)=c\left(1: E^{*}: s_{\beta}\left(\phi \cap w^{-1} \Phi^{+}\right)\right)=c\left(s_{\beta}: E^{*}: \phi \cap w^{-1} \Phi^{+}\right) .
$$

By Lemma 4.3,

$$
c\left(1: E^{*}: \phi \cap w^{-1} \Phi^{+}\right)+c\left(s_{\beta}: E^{*}: \phi \cap w^{-1} \Phi^{+}\right)=0 .
$$

Thus in this case also, the left hand side of (4.1) is zero unless $w^{-1} \alpha \equiv \alpha$.

Finally, suppose that $\alpha$ is a short simple root in a factor of type $B_{2 n+1}$. Then $\alpha$ is noncompact. Suppose that $\beta=w^{-1} \alpha$ is compact and fix $\phi \in \mathcal{T}_{n}\left(\Phi, w^{-1} \alpha\right)$. Since $\beta$ is compact it cannot occur in the factor of $\phi$ of type $A_{1}$. Thus it occurs in a factor of type $B_{2}$. Write $\phi=\phi_{1} \cup \phi_{2} \cup \phi_{3}$, where $\phi_{2}$ is the irreducible factor of $\phi$ containing $\beta$, $\phi_{3}$ is the irreducible factor of $\phi$ of type $A_{1}$, and $\phi_{1}$ is the union of all other irreducible factors. Then $\phi_{2} \cap w^{-1} \Phi^{+}=\left\{\beta, \beta_{2}, \beta_{2} \pm \beta\right\}$ and $\phi_{3} \cap w^{-1} \Phi^{+}=\left\{\beta_{3}\right\}$, where $\beta_{2}, \beta_{3}$ are short noncompact roots. Define $\phi^{\prime}=\phi_{1} \cup \phi_{2}^{\prime} \cup \phi_{3}^{\prime}$, where $\phi_{2}^{\prime} \cap w^{-1} \Phi^{+}=$ 
$\left\{\beta, \beta_{3}, \beta_{3} \pm \beta\right\}$ and $\phi_{3}^{\prime} \cap w^{-1} \Phi^{+}=\left\{\beta_{2}\right\}$. Then $\phi^{\prime}=s_{\delta} \phi \in \mathcal{T}_{n}\left(\Phi, w^{-1} \alpha\right)$ and $\phi^{\prime} \cap w^{-1} \Phi^{+}=s_{\delta}\left(\phi \cap w^{-1} \Phi^{+}\right)$, where $\delta=\beta_{2}-\beta_{3}$. Thus, by (3.7),

$$
\epsilon\left(\phi^{\prime}: w^{-1} \Phi^{+}\right)=-\epsilon\left(\phi: w^{-1} \Phi^{+}\right) .
$$

Further, $\beta$ is a good root for $\phi$ and $\phi^{\prime}$, and $\phi_{\beta}=\phi_{\beta}^{\prime}$. Thus, again using Lemma 4.3,

$$
\begin{aligned}
& c\left(1: E^{*}: \phi \cap w^{-1} \Phi^{+}\right)+c\left(1: E^{*}: \phi \cap w^{-1} s_{\alpha} \Phi^{+}\right) \\
= & c\left(1: E^{*}: \phi \cap w^{-1} \Phi^{+}\right)+c\left(s_{\beta}: E^{*}: \phi \cap w^{-1} \Phi^{+}\right), \\
& 2 c\left(1: E^{*}: \phi_{\beta} \cap w^{-1} \Phi^{+}\right)=2 c\left(1: E^{*}: \phi_{\beta}^{\prime} \cap w^{-1} \Phi^{+}\right) \\
= & c\left(1: E^{*}: \phi^{\prime} \cap w^{-1} \Phi^{+}\right)+c\left(1: E^{*}: \phi^{\prime} \cap w^{-1} s_{\alpha} \Phi^{+}\right) .
\end{aligned}
$$

Thus when $w^{-1} \alpha \not \equiv \alpha$, terms on the left hand of (4.1) can be grouped in pairs which cancel.

Let $\beta \in \Phi$. We will say that $\beta$ is of type I if $\beta$ is a long root in an irreducible factor of $\Phi$ of type $C_{n}, n \geq 1$. (Note this includes the cases that $\beta$ is in an irreducible factor of type $A_{1} \simeq C_{1}$ or is a long root in an irreducible factor of $\Phi$ of type $B_{2} \simeq C_{2}$.) Otherwise we will say that $\beta$ is of type II.

Lemma 4.5. Let $w \in W$. Then $w^{-1} \alpha \equiv \alpha$ if and only if $w \in W\left(\Phi_{\alpha}\right) W_{K} \cup$ $s_{\alpha} W\left(\Phi_{\alpha}\right) W_{K}$. Further, when $\alpha$ is of type $I, W$ is the disjoint union of $W\left(\Phi_{\alpha}\right) W_{K}$ and $s_{\alpha} W\left(\Phi_{\alpha}\right) W_{K}$. When $\alpha$ is of type II, $W\left(\Phi_{\alpha}\right) W_{K}=s_{\alpha} W\left(\Phi_{\alpha}\right) W_{K}$.

Proof. When $\alpha$ is of type I, $w^{-1} \alpha \equiv \alpha$ for all $w \in W$ since all long roots in irreducible factors of type $C_{n}, n \geq 1$, are noncompact. It is also easy to check in this case (see $\S 5$ ) that $W$ is the disjoint union of $W\left(\Phi_{\alpha}\right) W_{K}$ and $s_{\alpha} W\left(\Phi_{\alpha}\right) W_{K}$. Thus the result is true trivially.

Suppose that $\alpha$ is of type II and $w^{-1} \alpha \equiv \alpha$. We check case by case in $\S 5$ that $w^{-1} \alpha$ and $\alpha$ are conjugate via an element of $W_{K}$. That is, there is $w_{2} \in$ $W_{K}$ such that $w_{2} w^{-1} \alpha=\alpha$. This implies that $w_{2} w^{-1} \in W\left(\Phi_{\alpha}\right)$, so that $w \in$ $W\left(\Phi_{\alpha}\right) W_{K}$. Conversely, suppose that $w \in W\left(\Phi_{\alpha}\right) W_{K}$ and write $w=w_{1} w_{2}$, where $w_{1} \in W\left(\Phi_{\alpha}\right), w_{2} \in W_{K}$. Then $w^{-1} \alpha=w_{2}^{-1} \alpha \equiv \alpha$, since $W\left(\Phi_{\alpha}\right)$ fixes $\alpha$ and $W_{K}$ preserves compact and noncompact roots. Thus $w^{-1} \alpha \equiv \alpha$ if and only if $w \in W\left(\Phi_{\alpha}\right) W_{K}$ in this case. Finally, note that $w^{-1} s_{\alpha} \alpha=-w^{-1} \alpha \equiv \alpha$ if and only if $w^{-1} \alpha \equiv \alpha$. Thus $w \in W\left(\Phi_{\alpha}\right) W_{K}$ if and only if $s_{\alpha} w \in W\left(\Phi_{\alpha}\right) W_{K}$ in this case, so that $W\left(\Phi_{\alpha}\right) W_{K}=s_{\alpha} W\left(\Phi_{\alpha}\right) W_{K}$.

Lemma 4.6. Suppose that $w \notin W\left(\Phi_{\alpha}\right) W_{K} \cup s_{\alpha} W\left(\Phi_{\alpha}\right) W_{K}$. Then both sides of equation (4.1) are zero.

Proof. In this case both $w$ and $s_{\alpha} w$ are not elements of $W\left(\Phi_{\alpha}\right) W_{K}$. Thus $s\left(w: E^{*}: \Phi_{\alpha}^{+}\right)$and $s\left(s_{\alpha} w: E^{*}: \Phi_{\alpha}^{+}\right)$are both defined to be zero, and so the right hand side of (4.1) is zero. Further, by Lemma $4.5, w^{-1} \alpha \not \equiv \alpha$ in this case. Thus by Lemma 4.4 the left hand side of (4.1) is also zero.

Because of Lemma 4.6, it suffices to prove (4.1) in the case that $w \in W\left(\Phi_{\alpha}\right) W_{K} \cup$ $s_{\alpha} W\left(\Phi_{\alpha}\right) W_{K}$. In fact, since both sides of (4.1) are symmetric in $w, s_{\alpha} w$, we may as well assume that $w \in W\left(\Phi_{\alpha}\right) W_{K}$. Define

$$
c(\alpha, \Phi)= \begin{cases}1 & \text { if } \alpha \text { is of type I; } \\ 2 & \text { if } \alpha \text { is of type II. }\end{cases}
$$


Note that this is consistent with the notation $c(\alpha, \phi)$ for $\phi \in \mathcal{T}_{n}(\Phi, \alpha)$ used in Lemma 4.3 , since a good root $\alpha \in \phi$ is of type I if it is long and is of type II if it is short.

Lemma 4.7. Suppose $w=w_{1} w_{2}$ where $w_{1} \in W\left(\Phi_{\alpha}\right), w_{2} \in W_{K}$. Then

$$
\begin{gathered}
s\left(w: E^{*}: \Phi^{+}\right)+s\left(s_{\alpha} w: E^{*}: \Phi^{+}\right) \\
=c(\Phi) \sum_{\phi \in \mathcal{T}_{n}(\Phi, \alpha)} \epsilon\left(\phi: w_{1}^{-1} \Phi^{+}\right)\left[c\left(1: w_{2} E^{*}: \phi \cap w_{1}^{-1} \Phi^{+}\right)\right. \\
\left.+c\left(s_{\alpha}: w_{2} E^{*}: \phi \cap w_{1}^{-1} \Phi^{+}\right)\right]
\end{gathered}
$$

and

$$
\begin{gathered}
s\left(w: E^{*}: \Phi_{\alpha}^{+}\right)+s\left(s_{\alpha} w: E^{*}: \Phi_{\alpha}^{+}\right) \\
=c\left(\Phi_{\alpha}\right) c(\alpha, \Phi) \sum_{\psi \in \mathcal{T}_{n}\left(\Phi_{\alpha}\right)} \epsilon\left(\psi: w_{1}^{-1} \Phi_{\alpha}^{+}\right) c\left(1:\left(w_{2} E^{*}\right)_{\alpha}: \psi \cap w_{1}^{-1} \Phi_{\alpha}^{+}\right) .
\end{gathered}
$$

Proof. By Lemma 4.1, the constants $s\left(w: E^{*}: \Phi^{+}\right)$satisfy (3.2). Thus we have

$$
\begin{aligned}
& s\left(w_{1} w_{2}: E^{*}: \Phi^{+}\right)+s\left(s_{\alpha} w_{1} w_{2}: E^{*}: \Phi^{+}\right) \\
& =s\left(w_{1}: w_{2} E^{*}: \Phi^{+}\right)+s\left(s_{\alpha} w_{1}: w_{2} E^{*}: \Phi^{+}\right) .
\end{aligned}
$$

Now applying Lemma 4.2 in the case that $w=w_{1}$ and noting that $w_{1}^{-1} \alpha=$ $\alpha, w_{1}^{-1} s_{\alpha}=s_{\alpha} w_{1}^{-1}$, we get

$$
\begin{gathered}
s\left(w_{1}: w_{2} E^{*}: \Phi^{+}\right)+s\left(s_{\alpha} w_{1}: w_{2} E^{*}: \Phi^{+}\right) \\
=c(\Phi) \sum_{\phi \in \mathcal{T}_{n}(\Phi, \alpha)} \epsilon\left(\phi: w_{1}^{-1} \Phi^{+}\right)\left[c\left(1: w_{2} E^{*}: \phi \cap w_{1}^{-1} \Phi^{+}\right)\right. \\
\left.+c\left(1: w_{2} E^{*}: \phi \cap w_{1}^{-1} s_{\alpha} \Phi^{+}\right)\right] \\
=c(\Phi) \sum_{\phi \in \mathcal{T}_{n}(\Phi, \alpha)} \epsilon\left(\phi: w_{1}^{-1} \Phi^{+}\right)\left[c\left(1: w_{2} E^{*}: \phi \cap w_{1}^{-1} \Phi^{+}\right)\right. \\
\left.+c\left(s_{\alpha}: w_{2} E^{*}: \phi \cap w_{1}^{-1} \Phi^{+}\right)\right] .
\end{gathered}
$$

For the second equation, by definition,

$$
\begin{gathered}
s\left(w: E^{*}: \Phi_{\alpha}^{+}\right)=s\left(w_{1}:\left(w_{2} E^{*}\right)_{\alpha}: \Phi_{\alpha}^{+}\right) \\
=c\left(\Phi_{\alpha}\right) \sum_{\psi \in \mathcal{T}_{n}\left(\Phi_{\alpha}\right)} \epsilon\left(\psi: w_{1}^{-1} \Phi_{\alpha}^{+}\right) c\left(1:\left(w_{2} E^{*}\right)_{\alpha}: \psi \cap w_{1}^{-1} \Phi_{\alpha}^{+}\right) .
\end{gathered}
$$

If $\alpha$ is of type I, $w \in W\left(\Phi_{\alpha}\right) W_{K}$ implies that $s_{\alpha} w \notin W\left(\Phi_{\alpha}\right) W_{K}$, so that

$$
s\left(s_{\alpha} w: E^{*}: \Phi_{\alpha}^{+}\right)=0 .
$$

Since $c(\alpha, \Phi)=1$ in this case, the second equation holds.

If $\alpha$ is of type II, $c(\alpha, \Phi)=2$, so that we must show that

$$
\begin{aligned}
& s\left(s_{\alpha} w: E^{*}: \Phi_{\alpha}^{+}\right) \\
& \quad=c\left(\Phi_{\alpha}\right) \sum_{\psi \in \mathcal{T}_{n}\left(\Phi_{\alpha}\right)} \epsilon\left(\psi: w_{1}^{-1} \Phi_{\alpha}^{+}\right) c\left(1:\left(w_{2} E^{*}\right)_{\alpha}: \psi \cap w_{1}^{-1} \Phi_{\alpha}^{+}\right) .
\end{aligned}
$$

In this case $s_{\alpha} \in W\left(\Phi_{\alpha}\right) W_{K}$. Write $s_{\alpha}=u_{1} u_{2}$, where $u_{1} \in W\left(\Phi_{\alpha}\right)$ and $u_{2} \in W_{K}$. Note that $u_{1}^{-1} \alpha=\alpha$ and

$$
u_{2}^{-1} \alpha=u_{2}^{-1} u_{1}^{-1} \alpha=s_{\alpha} \alpha=-\alpha .
$$

Also

$$
s_{\alpha} w=s_{\alpha} w_{1} w_{2}=w_{1} s_{\alpha} w_{2}=\left(w_{1} u_{1}\right)\left(u_{2} w_{2}\right)
$$


is the decomposition of $s_{\alpha} w$ in $W\left(\Phi_{\alpha}\right) W_{K}$. Thus, by definition,

$$
\begin{gathered}
s\left(s_{\alpha} w: E^{*}: \Phi_{\alpha}^{+}\right)=s\left(w_{1} u_{1}:\left(u_{2} w_{2} E^{*}\right)_{\alpha}: \Phi_{\alpha}^{+}\right) \\
=c\left(\Phi_{\alpha}\right) \sum_{\psi \in \mathcal{T}_{n}\left(\Phi_{\alpha}\right)} \epsilon\left(\psi: u_{1}^{-1} w_{1}^{-1} \Phi_{\alpha}^{+}\right) c\left(1:\left(u_{2} w_{2} E^{*}\right)_{\alpha}: \psi \cap u_{1}^{-1} w_{1}^{-1} \Phi_{\alpha}^{+}\right) .
\end{gathered}
$$

Since $u_{2} \in W_{K}$, and $u_{2} \alpha=-\alpha$, it follows that $u_{2}: \Phi_{\alpha} \rightarrow \Phi_{\alpha}$ is an automorphism which preserves compact roots. Thus $u_{2} \mathcal{T}_{n}\left(\Phi_{\alpha}\right)=\mathcal{T}_{n}\left(\Phi_{\alpha}\right)$ and we can rewrite

$$
\begin{aligned}
& s\left(s_{\alpha} w: \tau: \Phi_{\alpha}^{+}\right) \\
& \quad=c\left(\Phi_{\alpha}\right) \sum_{\psi \in \mathcal{T}_{n}\left(\Phi_{\alpha}\right)} \epsilon\left(u_{2} \psi: u_{1}^{-1} w_{1}^{-1} \Phi_{\alpha}^{+}\right) c\left(1:\left(u_{2} w_{2} E^{*}\right)_{\alpha}: u_{2} \psi \cap u_{1}^{-1} w_{1}^{-1} \Phi_{\alpha}^{+}\right) .
\end{aligned}
$$

But by (3.8),

$$
\epsilon\left(u_{2} \psi: u_{1}^{-1} w_{1}^{-1} \Phi_{\alpha}^{+}\right)=\epsilon\left(u_{1} u_{2} \psi: w_{1}^{-1} \Phi_{\alpha}^{+}\right)=\epsilon\left(\psi: w_{1}^{-1} \Phi_{\alpha}^{+}\right)
$$

since $u_{1} u_{2} \psi=s_{\alpha} \psi=\psi$. Further, $\left(u_{2} w_{2} E^{*}\right)_{\alpha}=u_{2}\left(w_{2} E^{*}\right)_{\alpha}$ and so, using (3.6) for the automorphism $u_{2}: \Phi_{\alpha} \rightarrow \Phi_{\alpha}$,

$$
c\left(1:\left(u_{2} w_{2} E^{*}\right)_{\alpha}: u_{2} \psi \cap u_{1}^{-1} w_{1}^{-1} \Phi_{\alpha}^{+}\right)=c\left(1:\left(w_{2} E^{*}\right)_{\alpha}: \psi \cap u_{2}^{-1} u_{1}^{-1} w_{1}^{-1} \Phi_{\alpha}^{+}\right) .
$$

Again,

$$
u_{2}^{-1} u_{1}^{-1} w_{1}^{-1} \Phi_{\alpha}^{+}=s_{\alpha} w_{1}^{-1} \Phi_{\alpha}^{+}=w_{1}^{-1} \Phi_{\alpha}^{+}
$$

Lemma 4.8. Suppose that $\alpha$ is a good root of $\phi \in \mathcal{I}_{n}(\Phi, \alpha)$. Then $\phi_{\alpha} \in \mathcal{T}_{n}\left(\Phi_{\alpha}\right)$ unless $\alpha$ is a long root in an irreducible factor of $\Phi$ of type $C_{2 n+1}$, and is in an irreducible factor of $\phi$ of type $C_{2}$.

Proof. Since $\alpha$ is a good root of $\phi \in \mathcal{T}_{n}(\Phi, \alpha), \phi_{\alpha}$ is spanned by strongly orthogonal noncompact roots. Thus we need only check when $\phi_{\alpha}$ is a two-structure for $\Phi_{\alpha}$. This depends only on the irreducible factor of $\Phi$ containing $\alpha$, so we may as well assume that $\Phi$ is irreducible. If $\Phi$ is of type $A_{1}, D_{2 n}, E_{7}, E_{8}$, or $G_{2}$, then every $\phi \in \mathcal{T}(\Phi)$ is of type $A_{1}^{k}$, and $\phi_{\alpha}$ is of type $A_{1}^{k-1}$, which is a two-structure for $\Phi_{\alpha}$. If $\Phi$ is of type $B_{2 n}, C_{2 n}$, or $F_{4}$, then every $\phi \in \mathcal{T}(\Phi)$ is of type $B_{2}^{n}$ and $\phi_{\alpha}$ is of type $B_{2}^{n-1} \times A_{1}$, which is a two-structure for $\Phi_{\alpha}$. Suppose that $\Phi$ is of type $B_{2 n+1}$ or $C_{2 n+1}$. Then every $\phi \in \mathcal{T}(\Phi)$ is of type $B_{2}^{n} \times A_{1}$. If $\alpha$ is a long root in the $B_{2 n+1}$ case or a short root in the $C_{2 n+1}$ case, then $\Phi_{\alpha}$ is of type $B_{2 n-1} \times A_{1}$ or $C_{2 n-1} \times A_{1}$. Further, $\alpha$ must be in a factor of type $B_{2} \simeq C_{2}$ of $\phi$, so that $\phi_{\alpha}$ is of type $B_{2}^{n-1} \times A_{1} \times A_{1}$, which is a two-structure for $\Phi_{\alpha}$. Now suppose that $\alpha$ is the short simple root in the $B_{2 n+1}$ case or the long simple root in the $C_{2 n+1}$ case. Then $\Phi_{\alpha}$ is of type $B_{2 n}$ or $C_{2 n}$. If $\alpha$ is in the $A_{1}$ factor of $\phi$, then $\phi_{\alpha} \in \mathcal{T}\left(\Phi_{\alpha}\right)$ since it is of type $B_{2}^{n}$. However, if $\alpha$ is in a $B_{2}$ factor of $\phi$, then $\phi_{\alpha}$ is of type $B_{2}^{n-1} \times A_{1} \times A_{1}$. This is not a two-structure for $\Phi_{\alpha}$. However in the $B_{2 n+1}$ case, $\alpha$ is the short simple root and so is noncompact. It is not good in $\phi$ when it occurs in a $B_{2}$ factor. Thus the $C_{2 n+1}$ case gives the only exception.

Define

$$
\mathcal{T}_{n}(\Phi, \alpha)^{\prime}=\left\{\phi \in \mathcal{T}_{n}(\Phi, \alpha): \phi_{\alpha} \in \mathcal{T}_{n}\left(\Phi_{\alpha}\right)\right\}
$$

Suppose that $\alpha$ is not a good root of $\phi \in \mathcal{T}_{n}(\Phi, \alpha)$. Then $\phi_{\alpha}$ is not spanned by strongly orthogonal noncompact roots, so that it cannot be in $\mathcal{T}_{n}\left(\Phi_{\alpha}\right)$. Thus if 
$\phi \in \mathcal{T}_{n}(\Phi, \alpha)^{\prime}$, then $\alpha$ is a good root for $\phi$ so that $c(\alpha, \phi)$ is defined as in Lemma 4.3 .

Lemma 4.9. Let $w=w_{1} w_{2}$, where $w_{1} \in W\left(\Phi_{\alpha}\right), w_{2} \in W_{K}$. Then

$$
\begin{aligned}
& s\left(w: E^{*}: \Phi^{+}\right)+s\left(s_{\alpha} w: E^{*}: \Phi^{+}\right) \\
& \quad=c(\Phi) \sum_{\phi \in \mathcal{T}_{n}(\Phi, \alpha)^{\prime}} c(\alpha, \phi) \epsilon\left(\phi_{\alpha}: w_{1}^{-1} \Phi_{\alpha}^{+}\right) c\left(1:\left(w_{2} E^{*}\right)_{\alpha}: \phi_{\alpha} \cap w_{1}^{-1} \Phi_{\alpha}^{+}\right) .
\end{aligned}
$$

Proof. By Lemma 4.7,

$$
\begin{aligned}
& s\left(w: E^{*}: \Phi^{+}\right)+s\left(s_{\alpha} w: E^{*}: \Phi^{+}\right) \\
& =c(\Phi) \sum_{\phi \in \mathcal{T}_{n}(\Phi, \alpha)} \epsilon\left(\phi: w_{1}^{-1} \Phi^{+}\right)\left[c\left(1: w_{2} E^{*}: \phi \cap w_{1}^{-1} \Phi^{+}\right)\right. \\
& \left.+c\left(s_{\alpha}: w_{2} E^{*}: \phi \cap w_{1}^{-1} \Phi^{+}\right)\right] .
\end{aligned}
$$

If $\alpha$ is not a good root of $\phi$, then by Lemma 4.3,

$$
c\left(1: w_{2} E^{*}: \phi \cap w_{1}^{-1} \Phi^{+}\right)+c\left(s_{\alpha}: w_{2} E^{*}: \phi \cap w_{1}^{-1} \Phi^{+}\right)=0 .
$$

If $\alpha$ is a good root of $\phi$, then, again by Lemma 4.3,

$$
\begin{gathered}
c\left(1: w_{2} E^{*}: \phi \cap w_{1}^{-1} \Phi^{+}\right)+c\left(s_{\alpha}: w_{2} E^{*}: \phi \cap w_{1}^{-1} \Phi^{+}\right) \\
=c(\alpha, \phi) c\left(1:\left(w_{2} E^{*}\right)_{\alpha}: \phi_{\alpha} \cap w_{1}^{-1} \Phi_{\alpha}^{+}\right) .
\end{gathered}
$$

Further, by Lemma $4.8, \phi \in \mathcal{T}_{n}(\Phi, \alpha)^{\prime}$ unless $\alpha$ is a long root in an irreducible factor of $\Phi$ of type $C_{2 n+1}$, and is in an irreducible factor of $\phi$ of type $C_{2}$. In this case write $\phi=\phi_{1} \cup \phi_{2} \cup \phi_{3}$, where $\phi_{2}$ is the irreducible factor of $\phi$ containing $\alpha, \phi_{3}$ is the irreducible factor of $\phi$ of type $C_{1}$, and $\phi_{1}$ is the union of all other irreducible factors. Then

$$
\phi_{2} \cap w_{1}^{-1} \Phi^{+}=\left\{\alpha, \alpha_{2}, \frac{1}{2}\left(\alpha_{2} \pm \alpha\right)\right\}
$$

and $\phi_{3} \cap w_{1}^{-1} \Phi^{+}=\left\{\alpha_{3}\right\}$, where $\alpha_{2}, \alpha_{3}$ are long roots. Define $\phi^{\prime}=\phi_{1} \cup \phi_{2}^{\prime} \cup \phi_{3}^{\prime}$, where

$$
\phi_{2}^{\prime} \cap w_{1}^{-1} \Phi^{+}=\left\{\alpha, \alpha_{3}, \frac{1}{2}\left(\alpha_{3} \pm \alpha\right)\right\}
$$

and $\phi_{3}^{\prime} \cap w_{1}^{-1} \Phi^{+}=\left\{\alpha_{2}\right\}$. Then

$$
\phi^{\prime}=s_{\delta} \phi \in \mathcal{T}_{n}(\Phi, \alpha) \quad \text { and } \quad \phi^{\prime} \cap w_{1}^{-1} \Phi^{+}=s_{\delta}\left(\phi \cap w_{1}^{-1} \Phi^{+}\right),
$$

where $\delta=1 / 2\left(\alpha_{2}-\alpha_{3}\right)$. Thus, by (3.7),

$$
\epsilon\left(\phi^{\prime}: w_{1}^{-1} \Phi^{+}\right)=-\epsilon\left(\phi: w_{1}^{-1} \Phi^{+}\right) .
$$

Further, $\phi_{\alpha} \cap w_{1}^{-1} \Phi^{+}=\left(\phi_{1} \cap w_{1}^{-1} \Phi^{+}\right) \cup\left\{\alpha_{2}, \alpha_{3}\right\}=\phi_{\alpha}^{\prime} \cap w_{1}^{-1} \Phi^{+}$. Thus terms corresponding to the case that $\alpha$ is a long root in an irreducible factor of $\Phi$ of type $C_{2 n+1}$, and is in an irreducible factor of $\phi$ of type $C_{2}$, can be grouped in pairs which cancel. have

Finally, for $\phi \in \mathcal{T}_{n}(\Phi, \alpha)^{\prime}$, since $\alpha$ is a simple root for $w_{1}^{-1} \Phi^{+}$, by Lemma 3.3 we

$$
\epsilon\left(\phi: w_{1}^{-1} \Phi^{+}\right)=\epsilon\left(\phi_{\alpha}: w_{1}^{-1} \Phi_{\alpha}^{+}\right)
$$


For any root $\beta \in \Phi$, define

$$
\mathcal{T}(\Phi, \beta)^{\prime}=\left\{\phi \in \mathcal{T}(\Phi): \beta \in \phi \text { and } \phi_{\beta} \in \mathcal{T}\left(\Phi_{\beta}\right)\right\} .
$$

Lemma 4.10. If $\beta$ is any root of $\Phi$, then $\phi \mapsto \phi_{\beta}$ gives a bijection between $\mathcal{T}(\Phi, \beta)^{\prime}$ and $\mathcal{T}\left(\Phi_{\beta}\right)$. Further, $\phi \mapsto \phi_{\alpha}$ gives a bijection between $\mathcal{T}_{n}(\Phi, \alpha)^{\prime}$ and $\mathcal{T}_{n}\left(\Phi_{\alpha}\right)$.

Proof. We may as well assume that $\Phi$ is irreducible. By definition, if $\phi \in \mathcal{T}(\Phi, \beta)^{\prime}$, then $\phi_{\beta} \in \mathcal{T}\left(\Phi_{\beta}\right)$. Now fix $\psi \in \mathcal{T}\left(\Phi_{\beta}\right)$. Suppose that $\phi=\psi \cup\{ \pm \beta\}$ is a twostructure for $\Phi$. (This will always be the case when $\Phi$ is of type $A_{1}, D_{2 n}, E_{7}, E_{8}$, or $G_{2}$, since in these cases all $\phi \in \mathcal{T}(\Phi)$ are of type $A_{1}^{k}$.) Then clearly $\phi_{\beta}=\psi$. Further, if $\phi^{\prime} \in \mathcal{T}(\Phi, \beta)^{\prime}$ with $\left(\phi^{\prime}\right)_{\beta}=\psi$, then $\phi \subset \phi^{\prime}$. But all two-structures of $\Phi$ are isomorphic, so $\phi=\phi^{\prime}$.

Thus we can assume that $\psi \cup\{ \pm \beta\}$ is not a two-structure for $\Phi$. Then $\Phi$ is of type $B_{n}, C_{n}$, or $F_{4}$ and $\Phi_{\beta}$ is of type $B_{n-1}, B_{n-2} \times A_{1}, C_{n-1}, C_{n-2} \times A_{1}, C_{3}$, or $B_{3}$. In any of these cases, $\psi$ has at most 2 irreducible factors of type $A_{1}$, and if it has two, they are spanned by roots of different lengths. If $\psi$ has no irreducible factors of type $A_{1}$, then $\psi \cup\{ \pm \beta\}$ is a two-structure for $\Phi$. Thus in the case that $\psi \cup\{ \pm \beta\}$ is not a two-structure for $\Phi, \psi$ has a unique irreducible factor $\psi_{1}$ of type $A_{1}$ spanned by a $\operatorname{root} \beta^{\prime}$ of the same length as $\beta$, and $\beta, \beta^{\prime}$ span an irreducible factor $\phi_{1}$ of $\Phi$ of type $B_{2}$. Write $\psi=\psi_{0} \cup \psi_{1}$. Then $\phi=\psi_{0} \cup \phi_{1}$ is the unique two-structure of $\Phi$ such that $\phi_{\beta}=\psi$. This completes the proof of the first statement.

By definition, if $\phi \in \mathcal{T}_{n}(\Phi, \alpha)^{\prime}$, then $\phi_{\alpha} \in \mathcal{T}_{n}\left(\Phi_{\alpha}\right)$. Now suppose that $\psi \in$ $\mathcal{T}_{n}\left(\Phi_{\alpha}\right)$. By the first part, there is a unique $\phi \in \mathcal{T}(\Phi, \alpha)^{\prime}$ such that $\phi_{\alpha}=\psi$. To show that $\phi \in \mathcal{T}_{n}(\Phi, \alpha)^{\prime}$, we just need to show that $\phi$ is spanned by a strongly orthogonal collection of noncompact roots. Write $\phi=\phi_{0} \cup \phi_{1}$, where $\phi_{1}$ denotes the irreducible factor of $\phi$ containing $\alpha$. Then $\phi_{0} \subset \phi_{\alpha}$, so that it is spanned by strongly orthogonal noncompact roots. Now

$$
\phi_{1}= \begin{cases}\{ \pm \alpha\}, & \text { if } \phi_{1} \text { is of type } A_{1}, \\ \{ \pm \alpha, \pm \beta, \pm \beta \pm \alpha\}, & \text { if } \phi_{1} \text { is of type } B_{2} \text { and } \alpha \text { is short } \\ \{ \pm \alpha, \pm \beta, \pm 1 / 2(\beta \pm \alpha)\}, & \text { if } \phi_{1} \text { is of type } B_{2} \text { and } \alpha \text { is long, }\end{cases}
$$

and

$$
\phi_{\alpha}=\phi_{0} \cup \begin{cases}\emptyset, & \text { if } \phi_{1} \text { is of type } A_{1}, \\ \{ \pm \beta\}, & \text { if } \phi_{1} \text { is of type } B_{2} .\end{cases}
$$

Note that in the $B_{2}$ case, $\beta$ is in an irreducible factor of $\phi_{\alpha}$ of type $A_{1}$ and hence must be noncompact.

Suppose that $\alpha$ is compact. This occurs only when $\alpha$ is a short root in an irreducible factor of $\Phi$ of type $B_{2 n}, C_{n}$, or $F_{4}$. In these cases $\phi_{1}$ must be of type $B_{2}$. Since $\alpha$ is compact and $\beta$ is noncompact, $\alpha \pm \beta$ are strongly orthogonal noncompact roots spanning $\phi_{1}$. Now suppose that $\alpha$ is noncompact. In the $A_{1}$ case it spans $\phi_{1}$. In the $B_{2}$ case, if $\alpha$ is a long root, then so is $\beta$, and $\alpha, \beta$ are strongly orthogonal noncompact roots spanning $\phi_{1}$. The case that $\alpha$ is a short noncompact root in a $B_{2}$ factor cannot occur. This is because $\Phi$ would have to be of type $B_{2 n+1}$ to have a short noncompact good root $\alpha$. Then, as in the proof of Lemma 4.8, for $\phi \in \mathcal{T}(\Phi, \alpha), \phi_{\alpha} \in \mathcal{T}\left(\Phi_{\alpha}\right)$ implies that $\alpha$ is in an irreducible factor of type $A_{1}$ of $\phi$. 
Lemma 4.11. Let $\phi \in \mathcal{T}_{n}(\Phi, \alpha)^{\prime}$. Then

$$
c(\Phi) c(\alpha, \phi)=c\left(\Phi_{\alpha}\right) c(\alpha, \Phi) .
$$

Proof. As usual it is enough to check the case that $\Phi$ is irreducible. Suppose that $\Phi$ is of type $D_{2 n}, n \geq 2, E_{7}, E_{8}$, or $G_{2}$. Then $\Phi_{\alpha}$ is of type $D_{2 n-2} \times A_{1}, D_{6}, E_{7}$, or $A_{1}$ respectively. Every $\alpha$ is of type II, so that $c(\alpha, \Phi)=2$. Further, every irreducible factor of $\phi$ is of type $A_{1}$, so that $c(\alpha, \phi)=1$. Thus we must show that $c(\Phi)=2 c\left(\Phi_{\alpha}\right)$. This follows immediately by inspection from the table preceding Theorem 3.4. The proof in the other cases is given in $\S 5$.

Proof of Theorem 3.4. Because of Lemma 4.1, it is enough to show that the constants $s\left(w: E^{*}: \Phi^{+}\right)$satisfy the patching conditions for every $w \in W, E^{*} \subset E^{\prime}$, when $\alpha$ is a simple root for a good choice $\Phi^{+}$of positive roots. Because of Lemma 4.6 , it is enough to prove the patching conditions for $w=w_{1} w_{2} \in W\left(\Phi_{\alpha}\right) W_{K}$. Using Lemmas 4.7 and 4.9, we have

$$
\begin{gathered}
s\left(w_{1} w_{2}: E^{*}: \Phi^{+}\right)+s\left(s_{\alpha} w_{1} w_{2}: E^{*}: \Phi^{+}\right) \\
=c(\Phi) \sum_{\phi \in \mathcal{T}_{n}(\Phi, \alpha)^{\prime}} c(\alpha, \phi) \epsilon\left(\phi_{\alpha}: w_{1}^{-1} \Phi_{\alpha}^{+}\right) c\left(1:\left(w_{2} E^{*}\right)_{\alpha}: \phi_{\alpha} \cap w_{1}^{-1} \Phi_{\alpha}^{+}\right) ; \\
s\left(w_{1} w_{2}: E^{*}: \Phi_{\alpha}^{+}\right)+s\left(s_{\alpha} w_{1} w_{2}: E^{*}: \Phi_{\alpha}^{+}\right) \\
=c\left(\Phi_{\alpha}\right) c(\alpha, \Phi) \sum_{\psi \in \mathcal{T}_{n}\left(\Phi_{\alpha}\right)} \epsilon\left(\psi: w_{1}^{-1} \Phi_{\alpha}^{+}\right) c\left(1:\left(w_{2} E^{*}\right)_{\alpha}: \psi \cap w_{1}^{-1} \Phi_{\alpha}^{+}\right) .
\end{gathered}
$$

Using Lemma 4.10, it suffices to prove that for all $\phi \in \mathcal{T}_{n}(\Phi, \alpha)^{\prime}$,

$$
c(\Phi) c(\alpha, \phi)=c\left(\Phi_{\alpha}\right) c(\alpha, \Phi) .
$$

This is Lemma 4.11.

\section{Technical Lemmas}

We still need to check Lemmas 3.1, 3.3, and 4.5 for irreducible root systems of types $C_{n}, n \geq 1, B_{n}, n \geq 3, D_{2 n}, n \geq 2, E_{7}, E_{8}, F_{4}$, and $G_{2}$. We also need to check Lemma 4.11 for irreducible root systems of types $C_{n}, n \geq 1, B_{n}, n \geq 3$, and $F_{4}$.

The Case $\Phi=G_{2}$. Suppose that $\Phi$ is of type $G_{2}$. We will write

$$
\begin{gathered}
\Phi^{+}=\left\{\alpha_{i}, \beta_{i}: 1 \leq i \leq 3\right\}, \\
\Phi_{K}^{+}=\left\{\alpha_{3}, \beta_{3}\right\},
\end{gathered}
$$

where the $\alpha_{i}$ are short, the $\beta_{i}$ are long, and $\left\langle\alpha_{i}, \beta_{i}\right\rangle=0,1 \leq i \leq 3$. Clearly $\Phi_{\alpha_{i}}=\left\{ \pm \beta_{i}\right\}, \Phi_{\beta_{i}}=\left\{ \pm \alpha_{i}\right\}, 1 \leq i \leq 3$. Thus a root is good just in case it is noncompact. We can assume that the roots are numbered so that $\left\{\alpha_{1}, \beta_{2}\right\}$ is the set of simple roots for $\Phi^{+}$. Then $\Phi^{+}$is a good choice of positive roots, and every simple root for $\Phi^{+}$is good. This completes the proof of Lemma 3.1 in this case.

Because of (3.8), it is enough to prove Lemma 3.3 for the good choice $\Phi^{+}$given above. The only two-structures of $\Phi$ have positive roots $\phi_{i}^{+}=\left\{\alpha_{i}, \beta_{i}\right\}, 1 \leq i \leq 3$. $\Phi^{+}$is the lexicographic ordering with respect to the basis $\beta_{1}, \alpha_{1}$ of $\phi_{1}$. Thus, using 
the definition on $\left[\mathrm{K}\right.$, page 501], we have $\epsilon\left(\phi_{1}: \Phi^{+}\right)=1$. Now $\phi_{2}^{+}=s_{\alpha_{1}} s_{\beta_{2}} \phi_{1}^{+}$, so that by (3.7),

$$
\epsilon\left(\phi_{2}: \Phi^{+}\right)=\epsilon\left(\phi_{1}: \Phi^{+}\right)=1 .
$$

Thus for each two-structure $\phi$ containing a simple root of $\Phi^{+}$, we have $\epsilon\left(\phi: \Phi^{+}\right)=1$. For either simple root $\alpha, \Phi_{\alpha}$ is of type $A_{1}$, so that for the unique two-structure $\phi$ containing $\alpha, \epsilon\left(\phi_{\alpha}: \Phi_{\alpha}^{+}\right)=1$. This completes the proof of Lemma 3.3.

Suppose that $\alpha$ is a simple root for $\Phi^{+}$and $w \in W$ with $w^{-1} \alpha \equiv \alpha$. Then $\alpha$ and $w^{-1} \alpha$ are both noncompact and the same length. The reflection $s_{\alpha_{3}}$ interchanges $\alpha_{1}$ and $\alpha_{2}$ while the reflection $s_{\beta_{3}}$ interchanges $\beta_{1}$ and $\beta_{2}$. Further, $s_{\alpha_{3}} s_{\beta_{3}}=-1$, so that any two noncompact roots of the same length are conjugate via $W_{K}$. This completes the proof of Lemma 4.5.

The Other Irreducible Cases. Now suppose that $\Phi$ is irreducible of type $B_{n}, n \geq$ $3, C_{n}, n \geq 1, D_{n}, n=2 k \geq 4, E_{7}, E_{8}$, or $F_{4}$. In each case we can realize $\Phi$ as a root system in $\mathbf{R}^{n}$, where $n$ is the rank of $\Phi$, except in the case of $E_{7}$, where we take $n=8$. Write $n=2 k$ or $n=2 k+1$. Let $\Phi^{+}$be the positive system corresponding to the set $S(\Phi)$ of simple roots, where

$$
\begin{gathered}
S\left(B_{n}\right)=\left\{e_{1}-e_{2}, e_{2}-e_{3}, \ldots, e_{n-1}-e_{n}, e_{n}\right\} ; \\
S\left(C_{n}\right)=\left\{e_{1}-e_{2}, e_{2}-e_{3}, \ldots, e_{n-1}-e_{n}, 2 e_{n}\right\} ; \\
S\left(D_{n}\right)=\left\{e_{1}-e_{2}, e_{2}-e_{3}, \ldots, e_{n-1}-e_{n}, e_{n-1}+e_{n}\right\} ; \\
S\left(E_{7}\right)=\left\{e_{3}-e_{4}, e_{4}-e_{5}, e_{5}-e_{6}, e_{6}-e_{7}, e_{7}+e_{8}, e_{7}-e_{8},\right. \\
\left.\frac{1}{2}\left(e_{1}-e_{2}-e_{3}-e_{4}-e_{5}-e_{6}-e_{7}+e_{8}\right)\right\} ; \\
S\left(E_{8}\right)=\left\{e_{2}-e_{3}, e_{3}-e_{4}, \ldots, e_{6}-e_{7}, e_{7}+e_{8}, e_{7}-e_{8},\right. \\
\left.\frac{1}{2}\left(e_{1}-e_{2}-e_{3}-e_{4}-e_{5}-e_{6}-e_{7}+e_{8}\right)\right\} ; \\
S\left(F_{4}\right)=\left\{e_{2}-e_{3}, e_{3}-e_{4}, e_{4}, \frac{1}{2}\left(e_{1}-e_{2}-e_{3}-e_{4}\right)\right\} .
\end{gathered}
$$

Note that $E_{7}$ is realized as the set of roots in $E_{8}$ orthogonal to $e_{1}+e_{2}$.

We define compact and noncompact roots in $\Phi$ by specifying that every simple root is noncompact when $\Phi$ is of type $B_{2 k+1}, D_{2 k}, E_{7}$, or $E_{8}$. If $\Phi$ is of type $B_{2 k}, C_{n}$, or $F_{4}$, all long simple roots are noncompact and all short simple roots are compact. Thus $\Phi^{+}$satisfies the condition necessary to be a good choice of positive roots.

Lemma 5.1. With the choices of compact and noncompact simple roots above, $\Phi$ is spanned by strongly orthogonal noncompact roots.

Proof. We will need the following facts, which are easily checked in each case. Let $\Phi$ be an irreducible root system of type $B_{n}, n \geq 3, C_{n}, n \geq 1, D_{n}, n=2 k \geq 4, E_{7}, E_{8}$, or $F_{4}$, and let $S=S(\Phi)=\left\{\alpha_{1}, \ldots, \alpha_{r}\right\}, r=\operatorname{rank} \Phi$, be the set of simple roots for $\Phi^{+}$as above. Any $\gamma \in \Phi^{+}$can be written as $\gamma=\sum_{i=1}^{r} m_{i} \alpha_{i}$, where the $m_{i}, 1 \leq i \leq r$, are nonnegative integers. Let $\gamma_{1}$ be the longest root in $\Phi^{+}$, that is, the root for which $m\left(\gamma_{1}\right)=\sum_{i=1}^{r} m_{i}$ is maximal. Then $\gamma_{1}$ is a long root, and $m\left(\gamma_{1}\right)$ is odd. Further, if $\Phi$ is of type $B_{2 k}, C_{n}$, or $F_{4}$, and $\alpha_{i}$ is short, then $m_{i}$ is even. Thus $\gamma_{1}$ is a long noncompact root in $\Phi$. Finally, if $\Phi_{1}^{+}=\left\{\beta \in \Phi^{+}:\left\langle\beta, \gamma_{1}\right\rangle=0\right\}$, then $\Phi_{1}$ has rank $r-1$, and the simple roots $S_{1}$ for $\Phi_{1}^{+}$are a subset of $S$.

We prove the lemma by induction on the rank of $\Phi$. Let $\gamma_{1}$ be the longest root in $\Phi^{+}$, and define $\Phi_{1}^{+}, S_{1} \subset S$ as above. Then if $\Phi$ is of type $B_{2 k+1}, B_{2 k}, C_{n}, D_{2 k}, E_{7}$, $E_{8}$, or $F_{4}$, then $\Phi_{1}$ is of type $B_{2 k-1} \times A_{1}, B_{2 k-2} \times A_{1}, C_{n-1}, D_{2 k-2} \times A_{1}, D_{6}, E_{7}$, or $C_{3}$ respectively, where the $A_{1}$ factors are generated by long roots. Since $S_{1} \subset S$, 
every long root in $S_{1}$ is noncompact. Further, when $\Phi_{1}$ has an irreducible factor of type $B_{2 k-1}$, the short simple root comes from $B_{2 k+1}$ and is noncompact. When $\Phi_{1}$ has an irreducible factor of type $B_{2 k-2}$ or $C_{n-1}$, the short simple root comes from $\Phi$ of type $B_{2 k}, C_{n}$, or $F_{4}$, and hence is compact. Thus $\Phi_{1}^{+}$satisfies the condition necessary to be a good choice of positive roots. By the induction hypothesis, $\Phi_{1}^{+}$ is generated by a set $\left\{\gamma_{2}, \ldots, \gamma_{r}\right\}$ of strongly orthogonal noncompact roots. Now $\left\{\gamma_{1}, \ldots, \gamma_{r}\right\}$ generates $\Phi$. Since $\gamma_{1}$ is long and noncompact, it is a set of strongly orthogonal noncompact roots.

Lemma 5.2. Let $\alpha \in \Phi$. Then $\alpha$ is a good root if it satisfies one of the following.

(i) $\Phi$ is of type $B_{2 k+1}, D_{2 k}, E_{7}$, or $E_{8}$, and $\alpha$ is noncompact;

(ii) $\Phi$ is of type $B_{2 k}, C_{n}$, or $F_{4}$, and $\alpha$ is long and noncompact, or short and compact.

Proof. Let $\gamma_{i}, 1 \leq i \leq r$, be a set of strongly orthogonal noncompact roots spanning $\Phi$. We will use facts about expansions of roots in terms of the basis $\left\{\gamma_{1}, \ldots, \gamma_{r}\right\}$ from [K, page 513].

Assume first that $\Phi$ is not of type $B_{2 k+1}$. Then all the $\gamma_{i}$ 's are long roots. Let $\alpha$ be a long noncompact root. If $\alpha= \pm \gamma_{i}$ for some $1 \leq i \leq r$, then $\Phi_{\alpha}$ is generated by the $\gamma_{j}, j \neq i$. Otherwise, there is $I \subset\{1,2, \ldots, r\}$ with four elements such that

$$
\alpha=\frac{1}{2} \sum_{i \in I} \epsilon_{i} \gamma_{i}
$$

where $\epsilon_{i}= \pm 1, i \in I$. Assume for simplicity of notation that $I=\{1,2,3,4\}$. For each $i \in I$, let $s_{i}$ denote the reflection in the root $\gamma_{i}$. Since the $\gamma_{i}$ 's are long noncompact roots, $s_{p} s_{q} \alpha=\alpha-\epsilon_{p} \gamma_{p}-\epsilon_{q} \gamma_{q}$ is long and noncompact for any $1 \leq p \neq q \leq 4$. Now the roots

$$
\alpha_{1}=s_{3} s_{4} \alpha, \quad \alpha_{2}=s_{2} s_{4} \alpha, \quad \alpha_{3}=s_{2} s_{3} \alpha
$$

are all noncompact long roots, mutually orthogonal, and orthogonal to $\alpha$. Thus

$$
\left\{\alpha_{i}: 1 \leq i \leq 3\right\} \cup\left\{\gamma_{j}: 5 \leq j \leq r\right\}
$$

is a set of strongly orthogonal noncompact roots spanning $\Phi_{\alpha}$.

Now suppose that $\alpha$ is a short compact root. Since $\Phi$ has a short root and is not of type $B_{2 k+1}, \Phi$ is of type $B_{2 k}, C_{n}$, or $F_{4}$. Then there is $I \subset\{1,2, \ldots, r\}$ with two elements such that

$$
\alpha=\frac{1}{2} \sum_{i \in I} \epsilon_{i} \gamma_{i},
$$

where $\epsilon_{i}= \pm 1, i \in I$. Assume for simplicity of notation that $I=\{1,2\}$, and let $\alpha_{1}=s_{2} \alpha$. Then since $\alpha$ is compact, $\alpha_{1}=\alpha-\epsilon_{2} \gamma_{2}$ is noncompact and is orthogonal to $\alpha$. Now

$$
\left\{\alpha_{1}\right\} \cup\left\{\gamma_{j}: 3 \leq j \leq r\right\}
$$

is a set of strongly orthogonal noncompact roots spanning $\Phi_{\alpha}$.

Finally, suppose that $\Phi$ is of type $B_{2 k+1}$. Then exactly one of the $\gamma_{i}$ 's, say $\gamma_{r}$, is a short root. Suppose that $\alpha$ is a long noncompact root. If $\left\langle\alpha, \gamma_{r}\right\rangle=0$, then we can construct a basis of strongly orthogonal noncompact roots for $\Phi_{\alpha}$ as above. 
Suppose that $\left\langle\alpha, \gamma_{r}\right\rangle \neq 0$. Then there is $I \subset\{1,2, \ldots, r-1\}$ with two elements such that

$$
\alpha=\frac{1}{2} \sum_{i \in I} \epsilon_{i} \gamma_{i}+\epsilon_{r} \gamma_{r}
$$

where $\epsilon_{r}= \pm 1, \epsilon_{i}= \pm 1, i \in I$. Assume for simplicity of notation that $I=\{1,2\}$. Then

$$
\alpha_{1}=s_{r} \alpha=\alpha-2 \epsilon_{r} \gamma_{r}
$$

is a long noncompact root orthogonal to $\alpha$. Further,

$$
\alpha^{\prime}=\frac{1}{2} \sum_{i \in I} \epsilon_{i} \gamma_{i}=\alpha-\epsilon_{r} \gamma_{r}
$$

is a short compact root, and

$$
\alpha_{2}=s_{2} \alpha^{\prime}=\alpha^{\prime}-\epsilon_{2} \gamma_{2}
$$

is a short noncompact root orthogonal to $\alpha$ and $\alpha_{1}$. Thus $\left\{\alpha_{1}, \alpha_{2}\right\} \cup\left\{\gamma_{3}, \ldots, \gamma_{r-1}\right\}$ is a strongly orthogonal set of noncompact roots spanning $\Phi_{\alpha}$.

Suppose that $\alpha$ is a short noncompact root. If $\alpha= \pm \gamma_{r}$, then $\Phi_{\alpha}$ is spanned by $\gamma_{1}, \ldots, \gamma_{r-1}$. Otherwise, $\left\langle\alpha, \gamma_{r}\right\rangle=0$, and there is $I \subset\{1,2, \ldots, r-1\}$ with two elements such that

$$
\alpha=\frac{1}{2} \sum_{i \in I} \epsilon_{i} \gamma_{i}
$$

Assume for simplicity of notation that $I=\{1,2\}$. Since $\alpha$ is noncompact, $\alpha^{\prime}=s_{2} \alpha$ is compact and short. Let

$$
\alpha_{1}=\alpha^{\prime}+\gamma_{r}, \quad \alpha_{2}=\alpha^{\prime}-\gamma_{r}
$$

Then $\alpha_{1}$ and $\alpha_{2}$ are long noncompact roots, mutually orthogonal, and orthogonal to $\alpha$. Thus $\left\{\alpha_{1}, \alpha_{2}\right\} \cup\left\{\gamma_{3}, \ldots, \gamma_{r-1}\right\}$ is a strongly orthogonal set of noncompact roots spanning $\Phi_{\alpha}$.

This completes the proof of Lemma 3.1.

Proof of Lemma 3.3. Let $\Phi^{+}$be the good choice of positive roots with simple roots $S(\Phi)$ given above. Because of (3.8), it is enough to prove Lemma 3.3 for this standard choice $\Phi^{+}$. We define a two-structure $\phi_{0}$ as follows. For $1 \leq i \leq k$, let $\phi_{i}$ be the intersection of $\Phi$ with the linear span of $\left\{e_{2 i-1}, e_{2 i}\right\}$. Let $\phi_{k+1}$ be the intersection of $\Phi$ with the linear span of $\left\{e_{n}\right\}$ if $n=2 k+1$. If $n=2 k$ is even, we set $\phi_{k+1}=\emptyset$. Then

$$
\phi_{0}=\bigcup_{i=1}^{k+1} \phi_{i}
$$


is called the canonical two-structure for the positive roots $\Phi^{+}$. Define an ordered orthogonal basis $B(\Phi)$ for $\phi_{0}$ as follows.

$$
\begin{gathered}
B\left(C_{n}\right)=\left(2 e_{1}, 2 e_{2}, \ldots, 2 e_{n}\right) ; \\
B\left(B_{2 k}\right)=B\left(D_{2 k}\right)=B\left(E_{8}\right)=B\left(F_{4}\right) \\
=\left(e_{1}+e_{2}, e_{1}-e_{2}, \ldots, e_{2 k-1}+e_{2 k}, e_{2 k-1}-e_{2 k}\right) ; \\
B\left(B_{2 k+1}\right)=\left(e_{1}+e_{2}, e_{1}-e_{2}, \ldots, e_{2 k-1}+e_{2 k}, e_{2 k-1}-e_{2 k}, e_{2 k+1}\right) ; \\
B\left(E_{7}\right)=\left(e_{1}-e_{2}, e_{3}+e_{4}, e_{3}-e_{4}, \ldots, e_{7}+e_{8}, e_{7}-e_{8}\right) .
\end{gathered}
$$

This ordered basis satisfies the conditions of the definition in [K, page 501]. That is, it contains the positive root in each irreducible factor of type $A_{1}$ and the two long positive roots $\alpha_{1}, \alpha_{2}$ in each irreducible factor of type $B_{2}$. Further, $\alpha_{1}$ immediately precedes $\alpha_{2}$ in the ordering when the roots are numbered so that $\alpha_{1}-\alpha_{2}$ is twice a positive root. Now, $\Phi^{+}$is the lexicographic ordering determined by $B(\Phi)$. Thus $\epsilon\left(\phi_{0}: \Phi^{+}\right)=1$.

Suppose that $\alpha$ is a simple root for $\Phi^{+}$which is contained in $\phi_{0}$. Then $\psi_{0}=$ $\left(\phi_{0}\right)_{\alpha} \in \mathcal{T}\left(\Phi_{\alpha}\right)$. If $\alpha$ is a long root in $\phi_{0}$, then $\alpha \in B(\Phi)$, and we define an ordered basis $B\left(\Phi_{\alpha}\right)$ for $\psi_{0}$ by deleting $\alpha$ from $B(\Phi)$. Suppose that $\alpha$ is a short root in $\phi_{0}$, that is, a short root in an irreducible factor $\phi_{i}, 1 \leq i \leq k$, which is of type $B_{2}$. Let $\alpha^{\prime}$ be the short positive root in $\phi_{i}$ orthogonal to $\alpha$, and let $\beta_{1}, \beta_{2}$ denote the long positive roots in $\phi_{i}$. Then we define an ordered basis $B\left(\Phi_{\alpha}\right)$ for $\psi_{0}$ by deleting $\beta_{1}, \beta_{2}$ from $B(\Phi)$, and replacing them with $\alpha^{\prime}$. In either case $B\left(\Phi_{\alpha}\right)$ is a basis for $\psi_{0}$ satisfying the conditions in [K, page 591], and $\Phi_{\alpha}^{+}=\Phi_{\alpha} \cap \Phi^{+}$is the lexicographic ordering determined by $B\left(\Phi_{\alpha}\right)$. Thus

$$
\epsilon\left(\left(\phi_{0}\right)_{\alpha}: \Phi_{\alpha}^{+}\right)=1=\epsilon\left(\phi_{0}: \Phi^{+}\right) .
$$

Now suppose that $\alpha$ is a simple root that is not in $\phi_{0}$. It is easy to check by looking at the lists of simple roots $S(\Phi)$ above that there is a simple root $\alpha^{\prime}$ such that $\alpha^{\prime} \in \phi_{0}, \alpha$ and $\alpha^{\prime}$ have the same length, and $\left\langle\alpha, \alpha^{\prime}\right\rangle \neq 0$. Write $\sigma=s_{\alpha^{\prime}} s_{\alpha}$. Then $\alpha=\sigma \alpha^{\prime} \in \sigma \phi_{0}$. Since $\alpha$ and $\alpha^{\prime}$ are simple,

$$
\Phi^{+} \cap \sigma^{-1} \Phi^{-}=\{\alpha, \beta\},
$$

where $\beta=s_{\alpha} \alpha^{\prime}=\alpha+\alpha^{\prime}$. Since $\beta$ has the same length as $\alpha^{\prime}$, but is not orthogonal to $\alpha^{\prime}$, it cannot be in $\phi_{0}$. Further, $\alpha \notin \phi_{0}$ by assumption. Thus $\sigma \phi_{0}^{+} \subset \Phi^{+}$. Also, $\beta$ and $\alpha$ are not in $\Phi_{\alpha^{\prime}}$, so that $\sigma \Phi_{\alpha^{\prime}}^{+} \subset \Phi^{+}$. Thus

$$
\epsilon\left(\sigma \phi_{0}: \Phi^{+}\right)=\operatorname{det} \sigma=1
$$

and

$$
\epsilon\left(\left(\sigma \phi_{0}\right)_{\alpha}: \Phi_{\alpha}^{+}\right)=\epsilon\left(\left(\phi_{0}\right)_{\alpha^{\prime}}: \Phi_{\alpha^{\prime}}^{+}\right)=1,
$$

since

$$
\sigma^{-1}\left(\sigma \phi_{0}\right)_{\alpha}=\left(\phi_{0}\right)_{\alpha^{\prime}}
$$

and

$$
\sigma^{-1} \Phi_{\alpha}^{+}=\sigma^{-1}\left(\Phi_{\alpha} \cap \Phi^{+}\right)=\Phi_{\alpha^{\prime}} \cap \sigma^{-1} \Phi^{+}=\Phi_{\alpha^{\prime}}^{+} .
$$

Thus for each simple root $\alpha$ of $\Phi^{+}$we have found one two-structure $\phi_{1}$ such that $\alpha \in \phi_{1}, \psi_{1}=\left(\phi_{1}\right)_{\alpha} \in \mathcal{T}\left(\Phi_{\alpha}\right)$, and

$$
\epsilon\left(\phi_{1}: \Phi^{+}\right)=\epsilon\left(\psi_{1}: \Phi_{\alpha}^{+}\right)=1 .
$$


Now take an arbitrary $\phi \in \mathcal{T}(\Phi)$ such that $\alpha \in \phi$ and $\phi_{\alpha} \in \mathcal{T}\left(\Phi_{\alpha}\right)$. Then there is $\sigma \in W\left(\psi_{1}, \Phi_{\alpha}^{+}\right)$such that $\phi_{\alpha}^{+}=\sigma \psi_{1}^{+}$. Let $\phi_{2}$ be the irreducible factor of $\phi_{1}$ containing $\alpha$. Then

$$
\phi_{2}^{+}= \begin{cases}\{\alpha\}, & \text { if } \phi_{2} \text { is of type } A_{1} ; \\ \{\alpha, \beta, \beta \pm \alpha\}, & \text { if } \phi_{2} \text { is of type } B_{2} \text { and } \alpha \text { is short; } \\ \left\{\alpha, \beta, \frac{1}{2}(\beta \pm \alpha)\right\}, & \text { if } \phi_{2} \text { is of type } B_{2} \text { and } \alpha \text { is long. }\end{cases}
$$

Now $\sigma \psi_{1}^{+} \subset \Phi_{\alpha}^{+}$and $\sigma \alpha=\alpha$. Further, when $\phi_{2}$ is of type $B_{2}$ we have $\beta \in \psi_{1}^{+}$so that $\sigma \beta \in \Phi_{\alpha}^{+}$. Now since $\alpha$ is simple for $\Phi^{+}$, we have $\sigma c(\beta \pm \alpha)=c(\sigma \beta \pm \alpha) \in \Phi^{+}$, where $c=1$ if $\alpha$ is short and $c=1 / 2$ if $\alpha$ is long. Thus $\sigma \phi_{1}^{+} \subset \Phi^{+}$and

$$
\epsilon\left(\sigma \phi_{1}: \Phi^{+}\right)=\operatorname{det} \sigma=\epsilon\left(\phi_{\alpha}: \Phi_{\alpha}^{+}\right) \text {. }
$$

Finally, since $\sigma \alpha=\alpha,\left(\sigma \phi_{1}\right)_{\alpha}=\sigma\left(\phi_{1}\right)_{\alpha}=\phi_{\alpha}$. This implies that $\sigma \phi_{1}=\phi$ by Lemma 4.10 .

Proof of Lemma 4.5. Suppose that $\alpha$ is a simple root of type I. Thus $\Phi=C_{n}, n \geq 1$, and $\alpha=2 e_{n}$. Then $\Phi_{\alpha}=C_{n-1}$, so that $W\left(\Phi_{\alpha}\right)$ contains all sign changes except $s_{\alpha}$. $W_{K}$ contains all permutations, so that $W$ is the disjoint union of $W\left(\Phi_{\alpha}\right) W_{K}$ and $s_{\alpha} W\left(\Phi_{\alpha}\right) W_{K}$.

Suppose that $\alpha$ is a simple root of type II and $w^{-1} \alpha \equiv \alpha$ for $w \in W(\Phi)$. We must show that $\alpha$ and $w^{-1} \alpha$ are conjugate via $W_{K}$. Since $\alpha$ and $w^{-1} \alpha$ have the same length, it will suffice to show that $\alpha$ and $\beta$ are conjugate via $W_{K}$ if $\beta \equiv \alpha$ and has the same length as $\alpha$.

If $\alpha$ is compact, then $\Phi$ is of type $B_{2 k}, C_{n}$, or $F_{4}$, and $\alpha$ is short. $\Phi_{K}$ is of type $B_{k} \times D_{k}, A_{n-1}$, or $A_{1} \times C_{3}$ respectively, and when $\Phi=F_{4}$, the $A_{1}$ factor in $\Phi_{K}$ is spanned by a long root. Thus any two short compact roots are in the same simple factor of $\Phi_{K}$, and hence are conjugate via $W_{K}$.

Suppose that $\alpha$ is noncompact. Then $\Phi$ is of type $B_{n}, n \geq 3, D_{2 k}, k \geq 2, E_{7}, E_{8}$, or $F_{4}$, and in the case of $\Phi=B_{2 k}$ or $F_{4}, \alpha$ is long.

We first consider the case that $\Phi$ is of type $B_{n}, n \geq 3$. Here $\Phi_{K}$ is of type $B_{k} \times D_{n-k}$, where $B_{k}$ is spanned by the even indices and $D_{n-k}$ is spanned by the odd indices. Thus $W_{K}$ contains all permutations of even indices, all permutations of odd indices, all sign changes of even indices, and an even number of sign changes of odd indices. Let $s_{i}$ denote the sign change $e_{i} \mapsto-e_{i}$. Suppose $n=2 k+1$ is odd, and let $\alpha=e_{2 k+1}$ be the short simple root. If $\beta$ is any short noncompact root, then $\beta= \pm e_{2 j+1}$, where $0 \leq j \leq k$. The permutation that exchanges $e_{2 k+1}$ and $e_{2 j+1}$ is in $W_{K}$. Further, since $n \geq 3$, there is $0 \leq i \neq j \leq k$, and the product of the sign changes $s_{2 i+1} s_{2 j+1} \in W_{K}$. Thus in any case we can find $v \in W_{K}$ with $v e_{2 k+1}= \pm e_{2 j+1}$.

If $\alpha$ is a long simple root, then $\alpha=e_{i}-e_{i+1}$ and $\beta= \pm e_{j} \pm e_{k}$, where, since $\beta$ is noncompact, we can assume without loss of generality that $j \equiv i \bmod 2$ and $k \equiv i+1 \bmod 2$. Using $W_{K}$ we can permute the pairs $(i, j)$ and $(i+1, k)$. As above, there is an element of $W_{K}$ which changes the sign of the term with the odd index. But $W_{K}$ also contains all sign changes corresponding to even indices. Thus we can find $v \in W_{K}$ with $v\left(e_{i}-e_{i+1}\right)= \pm e_{j} \pm e_{k}$.

The final case is when $\Phi$ is of type $D_{2 k}, k \geq 2, E_{7}, E_{8}$, or $F_{4}$, and in the case of $\Phi=F_{4}, \alpha$ is long. Let $\beta$ be any noncompact root in $\Phi$ of the same length as $\alpha$. We will show that when $\Phi$ is of type $E_{7}, E_{8}$, or $F_{4}$, there is a root system $\Phi_{1} \subset \Phi$ which contains both $\alpha$ and $\beta$, is of type $D_{2 p}, p \geq 2$, , and is spanned by 
strongly orthogonal noncompact roots. Thus it will suffice to prove the result when $\Phi=D_{2 p}, p \geq 2$. Then $\Phi_{K}=D_{p} \times D_{p}$, where one $D_{p}$ factor is spanned by the even indices and the other is spanned by the odd indices. In this case $\alpha=e_{i} \pm e_{i+1}$ for some $1 \leq i \leq 2 p-1$, and $\beta= \pm e_{j} \pm e_{k}$ where $j \equiv i \bmod 2$ and $k \equiv i+1 \bmod 2$. Using $W_{K}$ we can permute the pairs $(i, j)$ and $(i+1, k)$. We can also change the signs of $e_{j}$ and $e_{k}$ as above, since there are at least two even indices and at least two odd indices.

It remains to produce the root system $\Phi_{1}$. If $\Phi$ is of type $F_{4}$, then $\alpha$ and $\beta$ are long, and we can take $\Phi_{1}$ to be the set of long roots in $\Phi$. Suppose that $\Phi$ is of type $E_{7}$ or $E_{8}$. Write $\alpha=\gamma_{1}$ and let $\left\{\gamma_{2}, \ldots, \gamma_{r}\right\}$ be a set of strongly orthogonal noncompact roots spanning $\Phi_{\alpha}$. Then $\left\{\gamma_{1}, \ldots, \gamma_{r}\right\}$ is a set of strongly orthogonal noncompact roots spanning $\Phi$. As in the proof of Lemma 5.2, either $\beta= \pm \gamma_{i}$ for some $i$, or $\beta=\frac{1}{2} \sum_{i \in I} \epsilon_{i} \gamma_{i}$ where $I$ consists of four indices. In either case, there are at least $r-5 \geq 2$ of the $\gamma_{i}$ 's which are orthogonal to both $\alpha$ and $\beta$. For simplicity of notation assume that $\gamma_{r-1}, \gamma_{r}$ are orthogonal to both $\alpha$ and $\beta$. When $\Phi$ is of type $E_{7}$, let $\Phi_{1}=\left\{\gamma \in \Phi:\left\langle\gamma, \gamma_{r}\right\rangle=0\right\}$. When $\Phi$ is of type $E_{8}$, let $\Phi_{1}=\left\{\gamma \in \Phi:\left\langle\gamma, \gamma_{i}\right\rangle=0, i=r-1, r\right\}$. In either case $\Phi_{1}$ is of type $D_{6}$, is spanned by strongly orthogonal noncompact roots, and contains both $\alpha$ and $\beta$.

Proof of Lemma 4.11. We need to check Lemma 4.11 for irreducible root systems of type $C_{n}, n \geq 2, B_{n}, n \geq 3$, and $F_{4}$.

Suppose that $\Phi$ is of type $C_{n}, n \geq 1$. When $\alpha=2 e_{n}$ is the long simple root, $\Phi_{\alpha}$ is of type $C_{n-1}$ and $\alpha$ is a long root in $\phi$ for all $\phi \in \mathcal{T}(\Phi)$. In this case we always have

$$
c(\Phi)=c\left(\Phi_{\alpha}\right)=c(\alpha, \phi)=c(\alpha, \Phi)=1
$$

When $\alpha=e_{i}-e_{i+1}$ is a short simple root, $\Phi_{\alpha}$ is of type $C_{n-2} \times A_{1}$ and if $\alpha \in \phi$, it must be a short root in an irreducible factor of type $C_{2}$. Thus we have

$$
c(\Phi)=c\left(\Phi_{\alpha}\right)=1 \text { and } c(\alpha, \phi)=c(\alpha, \Phi)=2
$$

Suppose that $\Phi$ is of type $B_{n}, n \geq 3$. If $\alpha=e_{n}$ is the short simple root, then $\Phi_{\alpha}$ is of type $B_{n-1}$. When $n=2 k$ is even, every $\phi \in \mathcal{T}_{n}(\Phi, \alpha)^{\prime}$ is of type $B_{2}^{k}$, so that $\alpha$ must be in an irreducible factor of type $B_{2}$ of $\phi$. In this case we have

$$
c(\Phi)=2^{k-1}=c\left(\Phi_{\alpha}\right) ; \quad c(\alpha, \Phi)=c(\alpha, \phi)=2 .
$$

Suppose that $n=2 k+1$ is odd. Then $\phi \in \mathcal{T}_{n}(\Phi, \alpha)^{\prime}$ is of type $B_{2}^{k} \times B_{1}$ and $\alpha$ is in the irreducible factor of type $B_{1}$ of $\phi$. In this case we have

$$
c(\Phi)=2^{k}, \quad c\left(\Phi_{\alpha}\right)=2^{k-1}, \quad c(\alpha, \Phi)=2, \quad c(\alpha, \phi)=1
$$

When $\alpha=e_{i}-e_{i+1}$ is a long simple root, $\Phi_{\alpha}$ is of type $B_{n-2} \times A_{1}$. Let $\phi \in \mathcal{T}_{n}(\Phi, \alpha)^{\prime}$. Then $\alpha$ must be a long root in an irreducible factor of type $B_{2}$. When $n=2 k$ is even we have

$$
c(\Phi)=2^{k-1}, \quad c\left(\Phi_{\alpha}\right)=2^{k-2}, \quad c(\alpha, \phi)=1, \quad c(\alpha, \Phi)=2
$$

When $n=2 k+1$ is odd we have

$$
c(\Phi)=2^{k}, \quad c\left(\Phi_{\alpha}\right)=2^{k-1}, \quad c(\alpha, \phi)=1, \quad c(\alpha, \Phi)=2
$$

Suppose that $\Phi$ is of type $F_{4}$. If $\alpha$ is a short simple root (compact) and $\phi \in$ $\mathcal{T}_{n}(\Phi, \alpha)^{\prime}$, then $\Phi_{\alpha}$ is of type $B_{3}$ and $\alpha$ is a short root in an irreducible factor of $\phi$ 
of type $B_{2}$, so

$$
c(\Phi)=2=c\left(\Phi_{\alpha}\right) ; \quad c(\alpha, \Phi)=c(\alpha, \phi)=2 .
$$

Suppose that $\alpha$ is a long simple root (noncompact) and $\phi \in \mathcal{T}_{n}(\Phi, \alpha)^{\prime}$. Then $\Phi_{\alpha}$ is of type $C_{3}$, and since $\alpha$ is a long root in $\phi$ we have

$$
c(\Phi)=2, \quad c\left(\Phi_{\alpha}\right)=1, \quad c(\alpha, \Phi)=2, \quad c(\alpha, \phi)=1 .
$$

\section{REFERENCES}

[G-K-M] M. Goresky, R. Kottwitz, and R. MacPherson, Discrete series characters and the Lefschetz formula for Hecke operators, Duke Math. J. 89 (1997), 477-554. CMP 98:01

[HC1] Harish-Chandra, Discrete series for semisimple Lie groups I, Acta Math., 113 (1965), 241-318. MR 36:2744

[HC2] Harish-Chandra, Harmonic analysis on real reductive groups I, J. Funct. Anal., 19 (1975), 104-204. MR 53:3201

[H1] R. Herb, Characters of averaged discrete series on semisimple real Lie groups, Pac. J. Math., 80 (1979), 169-177. MR 80h:22020

[H2] R. Herb, Fourier inversion and the Plancherel theorem for semisimple real Lie groups, Amer. J. Math., 104 (1982), 9-58. MR 84e:22013

[H3] R. Herb, Fourier inversion and the Plancherel theorem, (Proc. Marseille Conf. 1982), Lecture Notes in Math. Vol 880, Springer-Verlag, Berlin and New York, 1981, 197-210. MR 83f: 22013

[H4] R. Herb, Discrete series characters and Fourier inversion on semisimple real Lie groups, TAMS, 277 (1983), 241-261. MR 84h:22032

[H-W] R. Herb and J.A. Wolf, The Plancherel theorem for general semisimple groups, Compositio Math., 57 (1986), 271-355. MR 87h:22020

[K] A.W. Knapp, Representation Theory of Semisimple Groups, An Overview Based on Examples, Princeton U. Press, Princeton, N.J., 1986. MR 87j:22022

[K-Z] A.W. Knapp and G. Zuckerman, Classification of irreducible tempered representations of semisimple groups, Ann. of Math. 116 (1982), 389-501; 119 (1984), 639. MR 84h:22034a,b; MR 85e:22023

Department of Mathematics, University of Maryland, College Park, Maryland 20742

E-mail address: rah@math.umd.edu 